\title{
Evolution of Mercury-like orbits: A numerical study
}

\begin{abstract}
J. Warell, O. Karlsson, and E. Skoglöv
Institutionen för Astronomi och Rymdfysik, Uppsala Universitet, Box 515, 75120 Uppsala, Sweden

Received 28 August 2002 / Accepted 9 June 2003

Abstract. The orbital evolution of $\sim 30000$ objects has been studied numerically for time scales of up to $11 \mathrm{Myr}$ in order to investigate the dynamical importance of Mercury on objects with orbits similar to the planet's, as well as the existence of stable hermeocentric orbits. We find that Mercury, despite its small mass, exhibits a strong dynamical influence on objects with heliocentric Mercury-like orbits. The orbits of such objects may become temporarily and repeatedly Trojan-like for durations exceeding $20 \mathrm{kyr}$, exhibiting horseshoe orbits in the 1:1 resonance with periods of around 60 years. Objects with Mercury-like orbits may evolve dynamically to Venus crossers on time scales of 50-100 kyr by successive close encounters with Mercury which quasi-instantaneously increase the semimajor axis and cause subsequent capture in strong secular resonances, which force the eccentricity to high values. Initially, all particles were located in the $v_{1}$ and $v_{11}$ resonances which strongly affect their dynamical evolution. Only objects for which the semimajor axis and eccentricity were significantly increased due to close encounters with Mercury escaped these resonances. The effects on the orbital elements of close approaches within the Hill radius of Mercury are generally minor and their values are then unlikely to change significantly. The largest changes to the orbital elements at such events occur for Hill sphere traverse times of 2 days, when $a, e$ and $i$ may change by as much as $0.07 \mathrm{AU}, 0.13$ and $3.5^{\circ}$. However, secular resonances are generally considerably more efficient in affecting the orbital elements, particularly $v_{2}$ and $v_{12}$. We find retrograde and prograde hermeocentric orbits which are stable for durations in eccess of 4.5 Myr for initial semimajor axes $<30$ Mercury radii. Retrograde orbiters are stable for longer time scales than prograde. Collisions with Mercury occur for heliocentric objects, temporarily captured objects and for large hermeocentric orbits of both senses of motion. Gravitational scattering by Venus may constitute a possible route for transporting mercurian meteorites to Earth. We estimate the number of objects presently with orbits similar to that of Mercury and find that the current existence of such an object in the region has a low probability. We suggest an observational approach that would make the detection of hermeocentric objects larger than $1 \mathrm{~km}$ possible with a moderately sized telescope.
\end{abstract}

Key words. celestial mechanics - planets and satellites: individual: Mercury - minor planets, asteroids

\section{Introduction}

Dynamical studies of orbits similar to Mercury's are of interest to resolve the question of the present existence of objects in (and mode of transport into and out of) close heliocentric and hermeocentric orbits, as well as the structure and evolution of the early inner solar system. Objects may have reached such orbits either (i) as members of a heliocentric planet crossing population experiencing close approaches to the terrestrial planets with resulting dynamical evolution to Mercury-like orbits, (ii) as heliocentric members of debris swarms from Mercuryimpacting objects subsequently captured in a mean motion resonance at a close encounter with the planet, or (iii) as a result of early dissipative capture in hermeocentric orbits from heliocentric orbits wholly exterior or interior to that of the planet (e.g., from a putative Vulcanoid population). In the case of scenarios (i) and (iii) we may possibly expect that such object may exist today; in the first case the planet crossing population is constant in number, in that depletion through orbital decay and gravitational scattering is counterbalanced by

Send offprint requests to: J. Warell, e-mail: johan.warell@astro.uu.se replenishment through main-belt collisions and resonance transport into the inner solar system (e.g., Werner et al. 2002; Rabinowitz 1997, and references therein); and in the second case since the number density of objects in the inner solar nebula at the end of planetary accretion was very high and thus the probability of dissipative capture of small objects into close and stable hermeocentric orbits was non-negligible (Weidenschilling 1978). Leake et al. (1987) find that a considerable fraction of the total mass that impacted the terrestrial planets during the late heavy bombardment could have additionally impacted Mercury by a Vulcanoid population. In the case of scenario (ii) we would not expect the presence of such objects unless they are the result of a very recent and major impact or collision in the region, since the survival time scale near Mercury is of the order of a few Myr (Gladman et al. 1996; Evans \& Tabachnik 1999).

Given that Mercury has a small mass and an orbit with high eccentricity and inclination situated deep in the Sun's gravitational well, it is of interest to study if heliocentric objects may be significantly dynamically disturbed by the planet. In this work, we show that such gravitational scattering may result in Trojan type orbits, temporary capture orbits, or, in 
conjunction with the action of secular resonances, dynamical forcing to Venus approaching orbits. The possibility of Mercury Trojans has previously only been hinted at (Campins et al. 1996). Such orbital evolutionary scenarios constitute possible ways to remove or add objects in the Mercury-like orbital regime and subsequently evolve to Earth crossing orbits on time scales of a few to tens of Myr. This has previously been shown by Monte Carlo studies (Melosh \& Tonks 1993) and numerical integrations (Gladman et al. 1996). The efficiency of transfer of mercurian meteorites to Earth has been estimated to about $10^{-2}$ of that for martian meteorites (Wetherill 1984). The latter possibility is of interest as a mercurian meteorite could possibly exist in the meteorite collections, if so most probably as a misclassified member of the enstatite achondrites or anorthositic lunar meteorites (Love \& Keil 1995). A relationship between enstatite achondrites and Mercury has been suggested on the basis of the similarity of their optical spectra (Burbine et al. 2002), though ejectional debris would most probably display the spectral properties of a solid rock or breccia rather than a matured regolith.

We show by numerical integration that objects with small velocities relative to Mercury, initiated in heliocentric orbits with elements similar to that of the planet, may be temporarily captured for durations of 100 days or more via transfer through the Lagrangian points L1 and L2. Objects may also be injected into the 1:1 mean motion resonance with Mercury for durations in excess of 20000 years during which they execute horseshoe orbits with periods of the order of 60 years. We demonstrate the existence of hermeocentric orbits which are stable for $>4.5 \mathrm{Myr}$ for initial semimajor axes $<30$ Mercury radii and eccentricities $<0.5$ in retrograde sense, and to circular orbits of the same radius in prograde sense. If any such object larger than $1 \mathrm{~km}$ currently exists it should be detectable with a moderately sized telescope, and we propose an observational strategy to perform such a survey.

In Sect. 2 we describe the strategy and details of the integrations. Section 3 contains the main results. In Sect. 4 we estimate the number of objects with orbits similar to Mercury's and in Sect. 5 we evaluate the possibility of their observational detection. Finally, in Sect. 6 we discuss our findings and relate them to previous work by other authors.

\section{The integrations}

We used the 15 th order $n$-body integrator RADAU (Everhart $1985)$ with variable timestep to integrate orbits of massless objects subjected to the perturbations of the eight major planets. The source of elements for the major planets was Larsen et al. (2001) which are based on the JPL DE200/LE200 ephemeris. The different integrations made and the motivation behind them are described below.

In an initial preparatory integration, a large number $(\sim 30000)$ of orbital element and $X Y Z-\dot{X} \dot{Y} \dot{Z}$ positionvelocity vector sets were created. These were centered on, and widely distributed around, those for Mercury in order not to initially restrict the choise of orbits to a range too similar to that of Mercury. These objects were integrated for 100 days to determine what element ranges were required for objects to approach the planet. About $\sim 100$ objects were found to traverse Mercury's Hill radius $\left(\sim 1.47 \times 10^{-3} \mathrm{AU} \sim 220000 \mathrm{~km} \sim\right.$ $90 R_{\text {Merc }}$ ) for a duration exceeding 10 days. Their elements were used as a basis to establish element ranges for the main integration (integration 1).

Around the elements obtained from the preparatory integration a new set of 10000 was generated. These were distributed within the following ranges and were generated randomly throughout the given element limits with a constant distribution function:

$$
\left\{\begin{array}{l}
a=0.390 \pm 0.025 \mathrm{AU} \\
e=1.971 a-0.557 \pm 0.006 \\
\omega=28.5 \pm 10.5^{\circ} \\
i=-0.0452 \omega+8.318 \pm 0.55^{\circ} \\
\Omega=-0.4363 \omega+60.92 \pm 4^{\circ} \\
M=-0.3808 \omega+21.06 \pm 3^{\circ} .
\end{array}\right.
$$

This set symmetrically surrounds the osculating orbital elements of Mercury at the start of integration at epoch JD 2452120.5 (equinox J2000.0):

$$
\left\{\begin{array}{l}
a=0.3870986 \mathrm{AU} \\
e=0.2056337 \\
i=7.00486^{\circ} \\
\omega=29.1301^{\circ} \\
\Omega=48.3291^{\circ} \\
M=9.93115^{\circ} .
\end{array}\right.
$$

The integrations lasted $13203-16520$ years. The time, duration and closest distance of all approaches within the Hill sphere of Mercury were recorded.

From integration 1, 675 specifically interesting objects were selected for further study in integration 2 . These had spent a minimum of 10 days within the Hill sphere during at least one approach. The integration lasted 100000 years and had an output interval for rectangular coordinates and velocities of 50 years. For integration 3, a total of 17 particularly interesting objects were selected from the previous integration, whose close approaches to Mercury were extraordinarily long or numerous. The output interval was set to 1 day in order to study the dynamics of the approaches in detail. Integration 3 lasted 1226 years.

Additionally, in integration 2 a small number of objects were identified whose semimajor axes were consistently departing from that of Mercury, thus secularly approaching or departing from the Sun coincident with large oscillating variations in eccentricity. Twelve such objects were integrated further in time to investigate whether the motion would bring them into the Vulcanoid region or whether the increase in eccentricity would cause the objects to attain Venus approaching orbits. Integration 4 lasted $\sim 11$ Myr with a data output interval of 500 years.

Another interesting result of integration 2 was the presence of a hermeocentric retrograde object which was stable from the beginning throughout the full period of integration. A new longer integration, 5, was therefore made to study the long-term orbital stability of this object (the "original moon"), 
as well as a prograde object with the same initial velocity relative to Mercury but moving in the opposite direction, and a number of prograde/retrograde particle pairs with circular and eccentric orbits within the Hill sphere and motions initiated at their perihermia (cf. Table 3).

As to the possibility to confidently integrate the orbital evolution of particles in the innermost solar system, we found that the effect of chaos on the positions and velocities of the integrated objects dominates the orbital evolution with respect to general relativistic effects (GE). While the effect of the latter is possible to estimate, that of the former is not. The effect of chaos appears irrespective of the secular frequencies in the system, whether or not they are corrected for the small effect of GE. It can be shown that for an object whose elements differ maximally from Mercury's as dictated by the employed element limits given above, the pericenter shift relative to the planet amounts to at most $10 \mathrm{~km}$ for the time frame between passages within the Hill sphere of Mercury (typically $<1000$ years). Thus, chaotic effects dominate completely the results of the numerical integrations. Furthermore, since the effects of non-gravitational effects (Poynting-Robertson and Yarkovsky radiative drag forces, collisions) cannot be constrained for massless particles and may significantly change the orbits of small real objects in the $\sim 10^{6}$ year time scale of the longest integrations (e.g., Gladman et al. 1996; Vokhroulicky et al. 2000), we decided not to perform unmotivated corrections for GE and instead accept the statistical nature of the processes in the analysis of the integrations.

\section{Results}

\subsection{Resonances in the Mercury regime}

For the range of solar distances relevant in this study, most of the important mean motion resonances are related to Mercury. Of these, the 1:1 resonance is the most interesting (Sect. 3.3) and may affect objects whose semimajor axes are in the range $\sim 0.385-0.389 \mathrm{AU}$. (In the following, the 1:1 mean motion resonance with Mercury is occasionally termed 1:1Me, with corresponding notation for the other planets and mean motion resonances.)

Other mean motion resonances in the region are primarily of high orders. During the $11 \mathrm{Myr}$ integrations a typical object spends a few hundred occasions, up to tens of kyr long, in such resonances. For all identified mean motion resonances with Mercury, the order $p$ (the smallest integer number of complete orbits executed by Mercury during the same time frame as the particle executes an integer number of complete orbits) of the resonance was greater than 10 , about two thirds were of order $p=30$ or higher and the resonances were generally not of the type $(p-1): p$ or $(p+1)$ : $p$ (object:planet notation). Approximately $40 \%$ of the mean motion resonances encountered by an object were attributable to Mercury and the remaining due approximately equally to Venus, Earth and Mars (for the latter, only 9:1Ma was identified). The time periods spent in these resonances varied between a few hundred years up to a few hundred thousand years.
The single most important low-order resonance, next to $1: 1 \mathrm{Me}$, is $2: 1 \mathrm{Ve}$. It does however not dominate the dynamical evolution in any way since objects are only temporarily located in it and typically spend equal time in other nearby high-order resonances. The situation is complicated by the fact that some mean motion resonances almost coincide in $a$, e.g. the 13:4 resonance with the Earth and the 18:23 with Mercury are at $a=0.4558 \mathrm{AU}$, while the 2:1 with Venus is at $a=0.4557$ AU. Generally, we find that mean motion resonances are weak in the inner solar system and do not have primary dynamical importance.

The $v_{2}$ and $v_{12}$ resonances are the dominating secular resonances for $i<16^{\circ}$ and $a=0.4-0.5$ AU (Michel \& Froeschlé 1997; Michel 1997), and involve the precession frequencies of the longitude of perihelion $\varpi$ and the longitude of the node $\Omega$ of Venus, respectively. Some of the objects integrated in this study which fulfilled the above element constraints experienced an increase in $a$ through repeated close encounters with Mercury, a process that has a time scale of $\sim 10^{5}$ years or more, followed by a considerable increase of $e$. Such objects may become Venus crossing after a time scale of a few $\sim 10^{5}$ years (Sect. 3.2) and experience close approaches to or collisions with Venus within a time scale of $\sim 10^{6}$ years. An object may still have close encounters with Mercury after being orbitally evolved to Venus crossing, but for such orbits close approaches with Mercury are less frequent. This evolution may be due to a secular resonance, possibly $v_{2}$.

Initially, all integrated objects were situated in the $v_{1}$ and $v_{11}$ resonances with Mercury. The only objects which escaped from these resonances experienced a significant increase in $a$ and $e$ due to close approaches with Mercury prior to the escape. As all integrated objects were initiated with Mercurylike orbital elements, about $10 \%$ of them would collide with this planet within a time scale of $\sim 35000$ years since close encounters with Mercury are inefficient in increasing $a$ or $e$ to produce Venus approaching orbits. Likewise, it is equally unlikely that orbits evolve to Sun grazing and eventually cause objects to collide with the Sun through this process. However, a small number of objects will get trapped in secular resonances after experiencing a sufficient increase in $e$ or $a$ via a random walk-like process during a sequence of close encounters.

Even though it is demonstrated that particles with initially Mercury-like orbits may evolve to Venus approaching, we do not find in this work (almost certainly due to poor statistics) that any object subsequently obtains an Earth crossing orbit, which is necessary for the delivery of meteorites. In the $11 \mathrm{Myr}$ timeframe of integration 4 , one of the selected 12 objects $(8 \%)$ experienced a collision with Venus (Table 1), which is consistent with the results of Melosh \& Tonks (1993).

\subsection{Resonances: A case study}

We illustrate a possible scenario of dynamical evolution of a fictitious particle with an initial orbit similar to Mercury's with an 11 Myr long case study, when the integration was aborted. We also present five other objects summatically in Table 1, to 
Table 1. Initial orbital elements and evolution of case-study objects. Notes: Columns are, from left: Object designation; initial orbital elements (epoch is JD 2452 120.5); number of approaches within the Hill spheres of Mercury and Venus; time until achievement of Venus crossing orbit; time until first Venus close approach; lifetime; note ("CO" means collision). Data from integration 4.

\begin{tabular}{|c|c|c|c|c|c|c|c|c|c|c|c|c|}
\hline Object & $\begin{array}{l}a \\
(\mathrm{AU})\end{array}$ & $e$ & $\begin{array}{l}i \\
\left({ }^{\circ}\right)\end{array}$ & $\begin{array}{l}\omega \\
\left({ }^{\circ}\right)\end{array}$ & $\begin{array}{l}\Omega \\
\left({ }^{\circ}\right)\end{array}$ & $\begin{array}{l}M \\
\left({ }^{\circ}\right)\end{array}$ & $N_{\mathrm{Me}}$ & $N_{\mathrm{Ve}}$ & $\begin{array}{l}T_{\mathrm{VCO}} \\
(\mathrm{Myr})\end{array}$ & $\begin{array}{l}T_{\mathrm{VCA}} \\
(\mathrm{Myr})\end{array}$ & $\begin{array}{l}T_{\mathrm{int}} \\
(\mathrm{Myr})\end{array}$ & Note \\
\hline 38 & 0.3784229 & 0.1945032 & 7.20561 & 26.88380 & 47.82560 & 12.86873 & 1763 & 1 & 1.38 & 1.6 & 1.6 & Venus CO \\
\hline 60 & 0.3952884 & 0.2177328 & 6.96164 & 31.95132 & 43.96596 & 7.37471 & 1080 & 587 & 0.056 & 4.9 & 11.0 & Int. aborted \\
\hline 91 & 0.3651101 & 0.1603426 & 7.47004 & 26.93263 & 49.79761 & 12.39453 & 726 & 4502 & 0.076 & 4.9 & 11.0 & Int. aborted. Fig. 1 \\
\hline 96 & 0.3655905 & 0.1638854 & 6.80923 & 30.54178 & 44.94961 & 9.15255 & 2984 & 0 & - & - & 6.0 & Mercury CO \\
\hline 134 & 0.3775369 & 0.1924173 & 6.77262 & 23.02849 & 47.98355 & 11.55416 & 289 & 0 & - & - & 0.11 & Mercury CO \\
\hline
\end{tabular}

illustrate the widely disparate end states for initially similar particle velocities and positions.

The causes of orbital evolution were deciphered from the critical argument of the Kozai resonance (occurring when the circulation of $\omega$ ceases and $<\dot{\varpi}>=<\dot{\Omega}>$, thus the precession frequency $g$ of the longitude of perihelion equals the precession frequency $s$ of the node) which provides a temporary protection mechanism from close planetary encounters (Michel \& Thomas 1996), linear (i.e. involving only one planetary and one asteroid frequency) secular resonances with planets Mercury through Saturn in the longitude of perihelion $\varpi$ and ascending node $\Omega$ (e.g., Williams \& Faulkner 1981; Michel \& Froeschlé 1997), and mean motion resonances of order $p<30$ with the four inner planets which also act as (very) temporary protection mechanisms from close planetary encounters. The considered secular resonances occur for precession frequency relations of $\dot{\varpi}+2 j \dot{\omega}=g_{l}$ and $\dot{\Omega}+2 j \dot{\omega}=g_{1 l}$ where $l=1 \ldots 6$ and $j$ is an integer, and are termed the $v_{l}$ and $v_{1 l}$ resonances, respectively. These were studied through their critical arguments $\varpi-\varpi_{l}$ and $\Omega-\Omega_{l}$ which at resonant capture librate around $0^{\circ}$ and $180^{\circ}$.

The five objects in Table 1 are similar in that they were initially located in the secular resonances with the perihelion longitude and node of Mercury $\left(v_{1}\right.$ and $\left.v_{11}\right)$. Objects \#91 and \#60 were then transferred from the Mercury regime to Venus crossing orbits due to close approaches to Mercury which increased the semimajor axis, followed by subsequent capture in secular resonances. The semimajor axis of object \#96 was predominantly smaller than that of Mercury and was strongly affected by close encounters with the planet, leading to eventual collision. Object \#134 was initially locked in 1:1Me for a brief period and experienced no major changes in the elements throughout its evolution, which ended with a collision with Mercury after 114000 years. Object \#38 collided with Venus after a secular increase in $e$ similar to that experienced by the first two objects.

Below we describe in detail the orbital evolution of object \#91 to illustrate some of the complexity of possible dynamical evolution. The examples (Table 1) serve to illustrate (1) the wide variety of evolutionary paths possible from similar initial orbital elements, (2) the possibility of simultaneous location in overlapping linear secular and mean motion resonances, (3) the large number of impulsive planetary encounters which in most cases affect the particle orbits insignificantly, (4) the weak interaction with low-order mean motion resonances with the inner planets (of which 1:1Me and to a lesser extent $2: 1 \mathrm{Ve}$ appear to dominate), (5) the step-wise transition pattern in the evolution of semimajor axis, probably related to high-order mean motion resonances with single planets or linear combinations thereof, and (6) the dominance of secular resonances (mainly $v_{1}, v_{11}$ and $v_{5}$ ) in the dynamical evolution of the orbits.

Object \#91 (Fig. 1; note the change in time scale at $0.1 \mathrm{Myr}$ for the upper five panels) was initially located in the $v_{1}$ and $v_{11}$ secular resonances with Mercury though more strongly bound to the latter, as is indicated by smaller libration amplitude and longer capture duration. $a$ is quickly forced from 0.36 to 0.44 by the action of close approaches. Simultaneously with a capture in $v_{12}$ at 50-95 kyr, $e$ oscillates between 0.1 and 0.3 with a period of $\sim 20 \mathrm{kyr}$ while $i$ is stable. At $95 \mathrm{kyr}$ a series of temporally close approaches (within the Hill sphere) to Mercury occurs, which decreases $a$ and breaks the oscillation in $e$ to allow a capture in an unidentified secular resonance at $a=0.44 \mathrm{AU}, e=0.2$ and $i=8^{\circ}$. At the same time, $v_{1}$ and $v_{11}$ continue to influence the dynamical evolution until $\sim 150 \mathrm{kyr}$. The secular resonance forces the eccentricity to high values, 0.65 , until resonant behavior is broken at $820 \mathrm{kyr}$, probably due to a series of approaches to Mercury which act to increase $a$ by $\sim 0.004$ AU. From 1.5 to $2.1 \mathrm{Myr} e$ is again increasing followed by a significant increase in $i$ from 10 to $25^{\circ}$ during the period 1.9-2.0 Myr. After 2 Myr the particle enters a stable region in phase space with evolution similar to that of typical non-resonant main-belt asteroids. The eccentricity oscillates with two periods, approximately 0.4-0.8 Myr and $30 \mathrm{kyr}$, simultaneously with a slow secular increase. The inclination is constantly high, $20-25^{\circ}$, and subject to large oscillations with amplitudes of up to $10^{\circ}$. Close approaches within the Hill sphere of Venus, starting around 5 Myr, affect $a$ only marginally. Likewise, the importance of mean motion resonances in controlling the dynamical evolution is only marginal. Captures in $6: 1 \mathrm{Ma}, 3: 1 \mathrm{Ea}$ and $5: 7 \mathrm{Me}$ occur occasionally during periods of at most $200 \mathrm{kyr}$, but the strong 2:1 mean motion resonance with Venus is completely missed. The semimajor axis experiences a large number of step-wise transitions throughout the evolutionary time period, which we attribute to high-order mean motion resonances with the inner four planets interrupted by close approaches.

\subsection{Trojan-like orbits at the 1:1 mean motion resonance}

Due to the similarity of orbital elements to Mercury's for objects in integration 2, a majority develop temporary horseshoe 
Orbital evolution of object 91
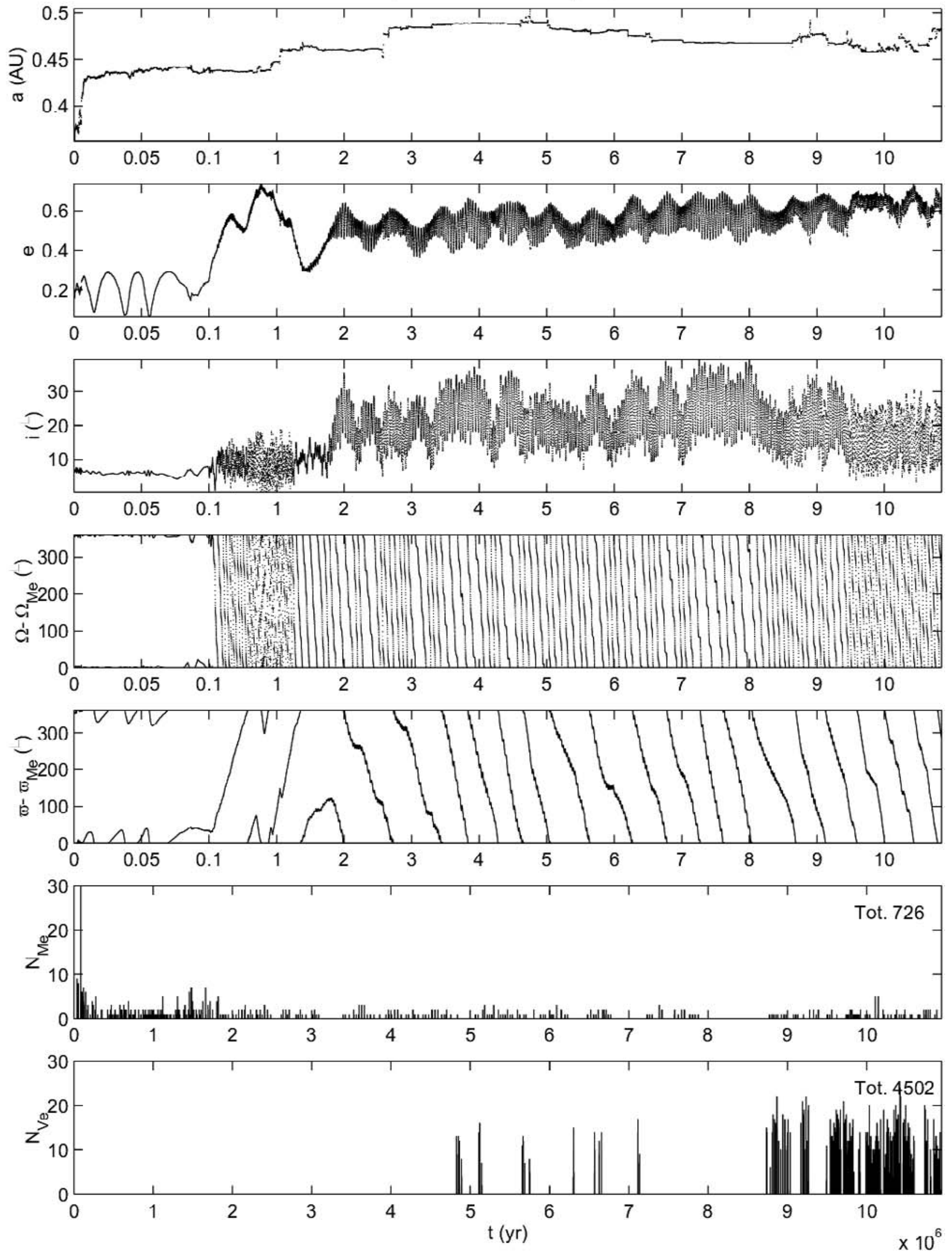

Fig. 1. Orbital evolution of heliocentric object \#91. The semi major axis, eccentricity, inclination, and critical arguments of the $v_{11}$ and $v_{1}$ resonances are plotted in panels one through five from top (note the change in time scale at $0.1 \mathrm{Myr}$ to accentuate the initial evolution; the tick step is $50 \mathrm{kyr}$ before and $1 \mathrm{Myr}$ after this time). The frequency of close approaches to Mercury and Venus are plotted in the lower two panels (bin size $1100 \mathrm{yr}$, uniform time scale). See Sect. 3.2 and Table 1 for details. 

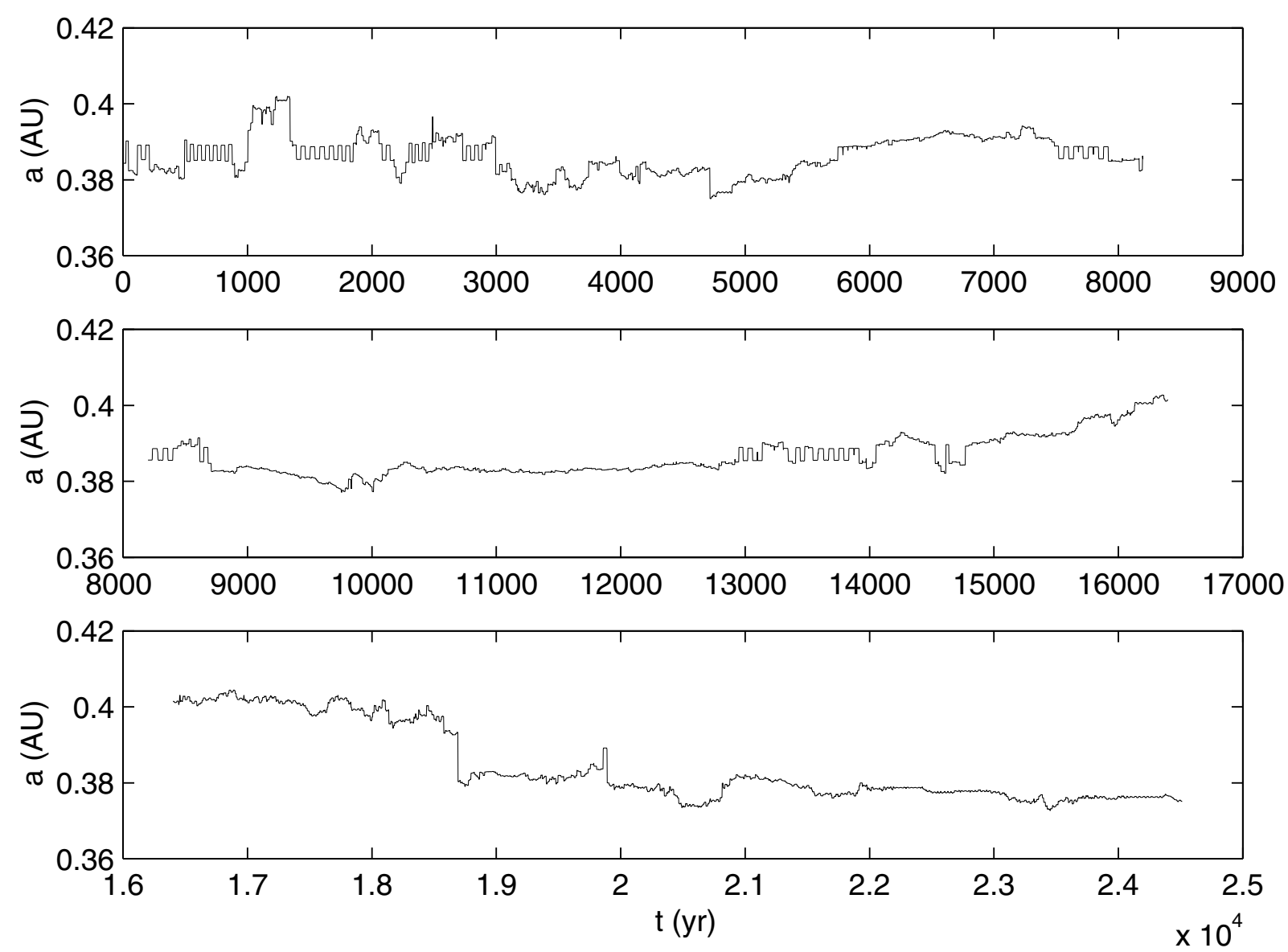

Fig. 2. Evolution of semimajor axis for a particle which repeatedly is locked in the $1: 1$ mean motion resonance with Mercury at 0.387 AU, during which it executes horseshoe orbits. During some capture events (e.g., 14.7 and $19.9 \mathrm{kyr}$ ), only a half horseshoe period is executed before ejection. At some close approaches to Mercury ( 7.7 and $13.9 \mathrm{kyr})$, the particle continues past the planet rather than performing a turn in mean longitude with respect to Mercury, as is ordinarily the case for a regular horseshoe orbit.

orbits which encompass both the L4 and L5 triangular libration points of the 1:1 mean motion resonance, similar to those of loosely bound Jupiter Trojan asteroids. As much as $72 \%$ of the objects experienced at least two consecutive direction changes in the mean longitude with respect to Mercury during the integration, corresponding to a half horseshoe period. One of these objects was trapped in a horseshoe orbit for $>23254$ years. None of the objects in our integrations experienced a tadpole orbit, which is typical for Jupiter Trojans tightly locked in the $1: 1$ resonance.

Based on 1500 years of integration of 675 objects, $18 \%$ of the Trojan objects remain in temporary horseshoe orbits for more than one full period. All such orbits are initiated and terminated at a close approach with Mercury, although not necessarily at a passage within the Hill sphere. The temporal evolution of the semimajor axis displays a square wave shape due to the fact that it is only at close approaches to Mercury that the planet's gravitational influence is strong enough to cause significant element change of the horseshoe orbit (unlike the case for Jupiter Trojans, which constantly adjust to the gravitational pull of Jupiter). A particle experiencing repeated and frequent 1:1 Me capture events is illustrated in Fig. 2.

The length of the 1:1 resonance period is controlled by the difference in semimajor axis between the librating object and Mercury, with libration period increasing for decreasing libration amplitude. The analytically estimated period for a tadpole Mercury Trojan is about 150 years (from Zhao $\&$ Liu 1992), while the longest periods found in this work are around 80 years for the deepest horseshoe orbits. A few objects with longer periods are evident in the numerical integration but they are not stable for more than the duration of one complete horseshoe orbit. Since the length of the libration period is not related to the number of orbital revolutions before ejection, strongly bound Mercury Trojans are not better protected against resonant ejection than weakly bound objects. The timing of ejection is effectively coupled to the geometrical circumstances at each approach to Mercury, such as the sense of the approach orbit and minimal approach distance. A statistical analysis shows that the importance of L4 and L5 for injection into and ejection out of the 1:1 resonance is equal.

\subsection{Close approaches and temporary captures}

For the particles selected for long-time investigation in integration 2, close approaches to Mercury, defined as passages within the Hill radius, are frequent. These objects had spent more than 10 consecutive days within the Hill sphere during the $\sim 15$ kyr duration of integration 1 . The average time span 

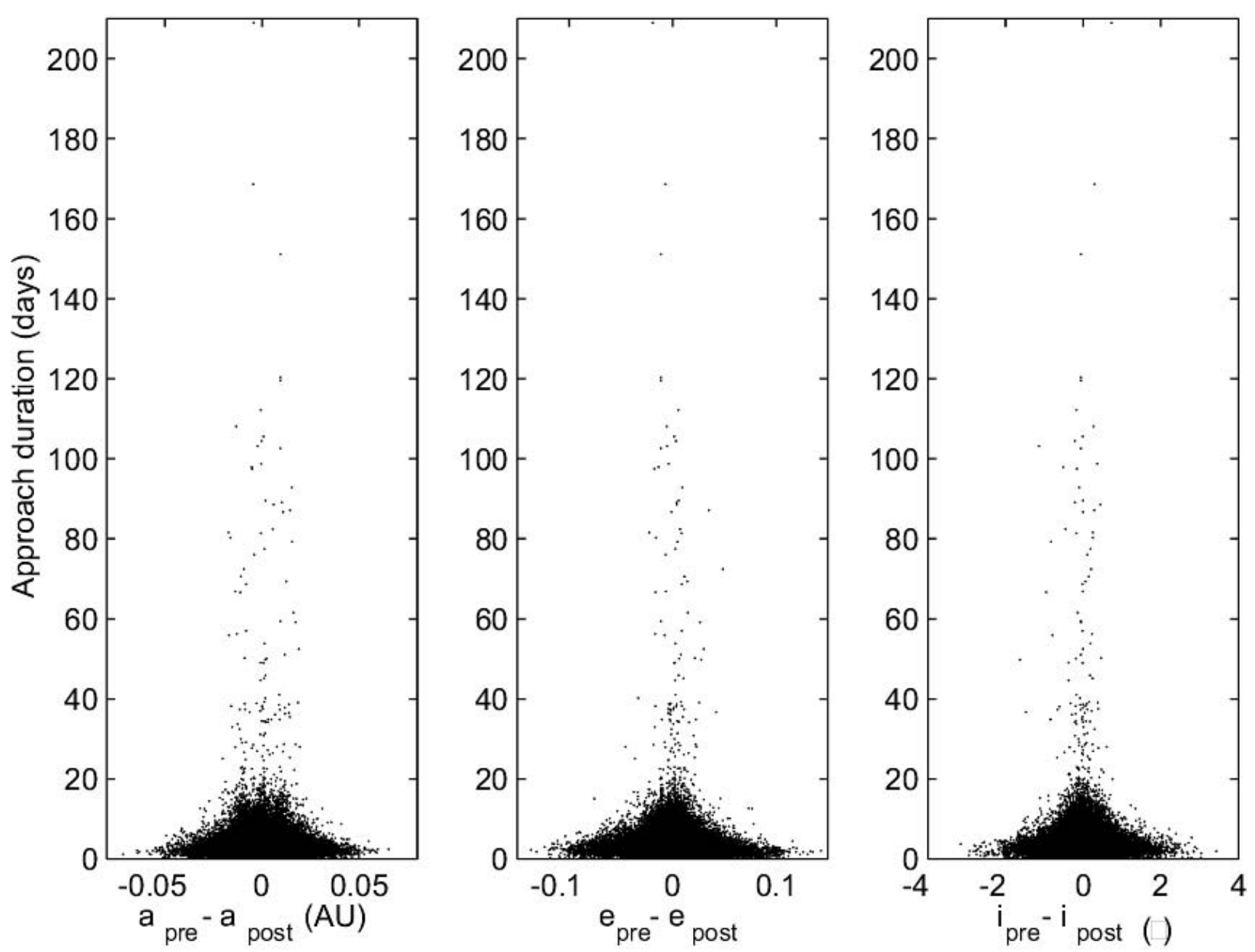

Fig. 3. Distribution of the changes in orbital element values versus approach duration for heliocentric objects performing close approaches within the Hill sphere Mercury. The largest element changes occur for durations of about 2 days, while more long-lasting temporary capture events cause insignificant changes.

between such approaches for a given object is 286 years with a median of 215 years. The average duration $t$ of a close approach, during which the object is continually within the Hill sphere, is 4.0 days with a median value of 3.3 days and a recorded maximum duration of 208.9 days.

Close encounters with Mercury are comparatively ineffective in changing the orbital elements of approaching objects. Changes are maximal for approach durations of about 2 days and reach maximum values of $\Delta a=0.07 \mathrm{AU}, \Delta e=0.13$ and $\Delta i=3.5^{\circ}$ (Fig. 3). The smallest changes occur for objects passing through the outer regions of the Hill sphere, for which $t<2$ days, as well as for objects with very deep approaches ( $t>15$ days) which perform a significant part of a full trajectory around Mercury.

The distribution of approach duration versus $\Delta a$ shows three distinctly separated peaks at about $-0.01,0$ and $0.01 \mathrm{AU}$ (Fig. 3, left panel). The extreme $\Delta a$ peaks may be attributable to the existence of Mercury Trojans which perform a turn in mean longitude in the planetary corotating frame. Such objects experience shifts in semimajor axis of $\sim 0.005-0.01 \mathrm{AU}$, all of which are greater than $0.002 \mathrm{AU}$. The central peak may be due to objects which do not perform a turn in mean longitude relative to Mercury at approach (i.e., $a_{\text {pre }} \sim a_{\text {post }}$ ). For the object illustrated in Fig. 2, such events occur at 7.7 and 13.9 kyr.

The weak gravitational disturbance by Mercury is illustrated in Fig. 4, where pre- and post-approach values for individual elements are compared. Generally the elements do not change significantly and therefore scatter along the $1: 1$ symmetry line, preferentially situated around the value for Mercury. The mean anomaly plane is homogeneously filled, reflecting the fact that there is no correlation between the positions of a particle in its orbit before and after a close approach. The semimajor axis plane is more complex. The preferentially populated, positively sloped, line defines the initial selection of semimajor axis values, and remains well populated as the probability of significant element scatter at close approach is small. The poorly occupied horizontal region defines the values of $a$ at injection into the 1:1 resonance, with the right half indicating entries from the direction of L4 and the left entries from the direction of L5. The poorly occupied vertical region defines the values of $a$ at ejection out of the resonance, with the lower half indicating ejections from L4 and the upper ejections from L5. The intersection of these two regions marks the phase space of objects which have missed a turning point or approached 

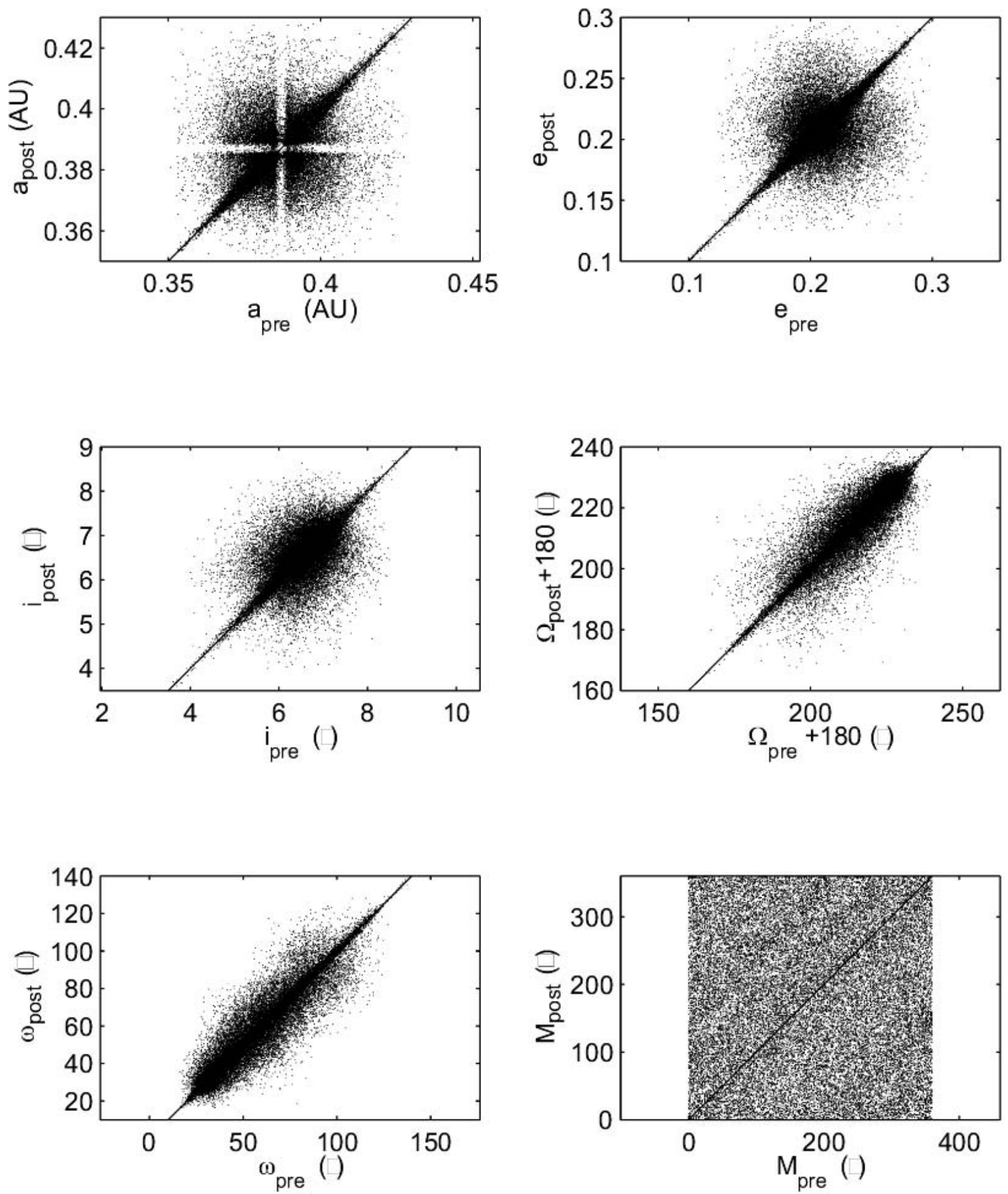

Fig. 4. Distributions of element changes pre versus post close encounters with Mercury. The 1:1 correspondence is indicated by a straight line, around which the distributions are symmetric. See Sect. 3.4 for further discussion.

Mercury at a turning event, passed within the Hill sphere and remained in the 1:1 resonance.

A selection of interesting temporary capture events are described in Table 2. These may be divided into two groups: (I) where the objects perform a deep passage inside the Hill sphere without performing a complete revolution, and (II) where the objects are temporarily captured and per- form at least one complete revolution in the same sense about Mercury. Objects in group II conform to the strict definition of a temporarily captured satellite set by Rickman \& Malmort (1981), for which the object is gravitationally bound to the planet, i.e., has an elliptically osculating planetocentric orbit (negative planetocentric orbital energy $E_{\text {orb}}$ ). Mercury exerted 
Table 2. Examples of temporary capture orbits. Columns are, from left: Object and capture event designation; group membership; period between first and last entry of Hill sphere ( $\left.t_{\text {orb }}\right)$; orbital elements $a, e$, and $i 100$ days prior to (first) entry of Hill sphere; change in orbital elements $a, e$, and $i$ (values at 100 days prior to (first) entry of Hill sphere minus values at 100 days post (last) exit of Hill sphere; mean anomaly at capture ( $M_{\text {cap }}$, first entry of Hill sphere) and escape ( $M_{\text {esc }}$, last exit of Hill sphere); number of complete revolutions in same sense between (first) entry and (last) escape of Hill sphere, relative to initial mean anomaly in hermeocentric orbit $\left(N_{\text {orb }}\right)$; rotational sense (retrograde or prograde) of each initiated revolution (temporally from left to right) relative to initial mean anomaly in hermeocentric orbit and to plane of orbit in the Mercury centered reference frame; average period of revolution $\left(<P_{\text {orb }}>\right)$; minimum distance to Mercury $\left(R_{\text {min }}\right.$ Mercury radii); and minimal energy of elleptic orbit relative to Mercury $\left(E_{\min }\right)$. Data from integration 3.

\begin{tabular}{|c|c|c|c|c|c|c|c|c|c|c|c|c|c|c|c|c|}
\hline $\begin{array}{r}\text { Object } \\
\& \text { event }\end{array}$ & Group & $\begin{array}{l}t_{\text {orb }} \\
\text { (d) }\end{array}$ & $\begin{array}{r}a_{\text {pre }} \\
(\mathrm{AU}) \\
\end{array}$ & $e_{\text {pre }}$ & $\begin{array}{l}i_{\text {pre }} \\
\left(^{\circ}\right) \\
\end{array}$ & $\begin{array}{r}\Delta a \\
(\mathrm{AU}) \\
\end{array}$ & $\Delta e$ & $\begin{array}{l}\Delta i \\
\left(^{\circ}\right)\end{array}$ & $\begin{array}{r}M_{\text {cap }} \\
\left({ }^{\circ}\right) \\
\end{array}$ & $\begin{array}{r}M_{\mathrm{esc}} \\
\left({ }^{\circ}\right) \\
\end{array}$ & $N_{\text {orb }}$ & Sense & $\begin{array}{r}<P_{\text {orb }}> \\
\text { (d) }\end{array}$ & $\begin{array}{r}R_{\min } \\
\left(R_{\mathrm{Merc}}\right) \\
\end{array}$ & $\begin{array}{r}E_{\min } \\
\left(\mathrm{AU}^{-1}\right) \\
\end{array}$ & Note \\
\hline 13.5 & II & 54.7 & 0.391 & 0.208 & 6.971 & -0.01 & -0.02 & 0.08 & 45 & 275 & 2.6 & RRR & 21.0 & 1.6 & -1480 & \\
\hline 13.5 & I & 11.6 & 0.379 & 0.193 & 7.051 & 0.01 & 0.02 & -0.09 & 321 & 12 & 0.6 & $\mathrm{P}$ & 19.3 & 10.6 & -898 & \\
\hline 13.5 & II & 32.0 & 0.391 & 0.201 & 6.997 & 0.01 & 0.02 & 0.07 & 208 & 257 & 1.1 & PP & 29.0 & 3.1 & -1210 & \\
\hline 138.1 & I & 7.3 & 0.381 & 0.195 & 6.808 & -0.00 & -0.00 & 0.07 & 353 & 25 & 0.5 & $\mathrm{P}$ & 14.6 & 18.4 & -290 & \\
\hline 238.2 & I & 34.0 & 0.394 & 0.216 & 6.993 & -0.00 & -0.02 & 0.17 & 332 & 112 & 1.0 & $\mathrm{P}$ & 34.0 & 16.7 & -621 & \\
\hline 769.8 & II & 87.5 & 0.379 & 0.194 & 6.972 & 0.00 & 0.00 & 0.07 & 96 & 214 & 5.5 & PPPPPR & 15.9 & 1.7 & -1910 & \\
\hline 256.10 & II & 66.9 & 0.390 & 0.211 & 6.805 & -0.01 & 0.00 & 0.15 & 274 & 286 & 2.3 & RRR & 29.1 & 14.0 & -410 & \\
\hline 853.3 & II & 143.5 & 0.397 & 0.213 & 7.154 & -0.01 & -0.02 & -0.16 & 267 & 206 & 4.1 & PPRPP & 35.0 & 6.5 & -1400 & Fig. 6 \\
\hline 628.1 & II & 80.0 & 0.377 & 0.190 & 7.105 & 0.02 & 0.0252 & -0.24 & 216 & 292 & 3.4 & PPPP & 23.5 & 10.2 & -1550 & \\
\hline 628.1 & II & 86.5 & 0.397 & 0.222 & 6.877 & -0.02 & -0.03 & 0.01 & 218 & 301 & 3.4 & PPPP & 25.4 & 15.9 & -1540 & \\
\hline 51.2 & I & 33.9 & 0.390 & 0.200 & 6.723 & -0.01 & -0.00 & 0.47 & 150 & 31 & 0.6 & $\mathrm{P} \rightarrow \mathrm{R}$ & 56.5 & 46.0 & -507 & Fig. 5 \\
\hline
\end{tabular}



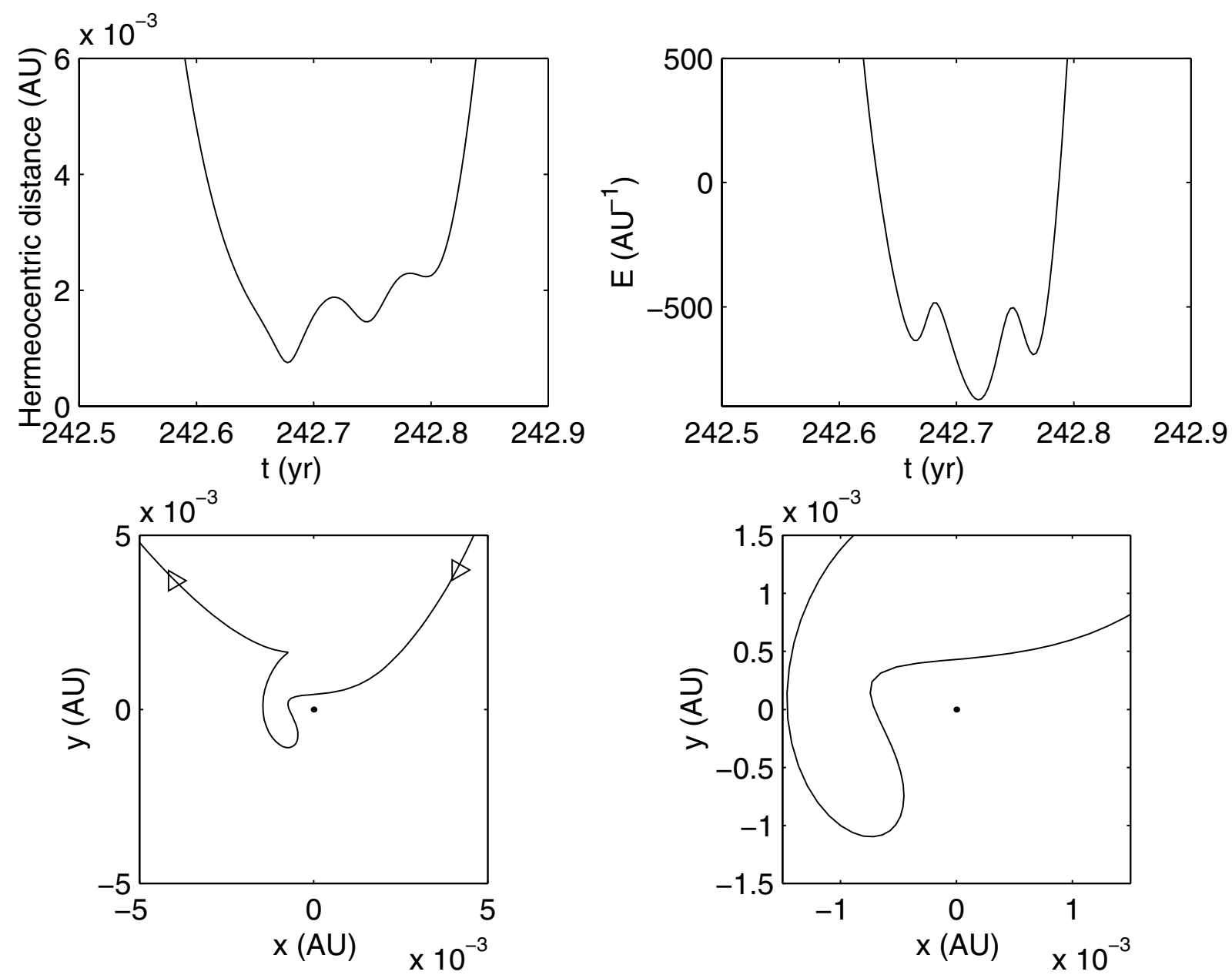

Fig. 5. Trajectory for a temporary capture of object 51.2. Entry occurs from the direction of L4 in a horseshoe orbit. For this group II member, an incomplete revolution including a change in directional sense of motion is executed. The upper left panel shows distance to Mercury, the upper right panel the orbital energy of the hermeocentric orbit (negative values indicating gravitational binding to the planet), and the two lower panels the orbit in the mercurian corotating system with Mercury at the center and the Sun on the negative $x$ axis, projected from above on the osculating $X-Y$ plane of Mercury. Direction of motion is indicated by arrows in the lower left panel. L1 and L2 are located on the $y$ axis at $x \sim \pm 1.5 \times 10^{-3} \mathrm{AU}$ and mark the radius of the Hill sphere (cf. Table 2).

a gravitational attraction on the object that dominated over the Sun's in all cases studied but one.

The distance to Mercury, the orbital energy and the trajectory in the mercurian corotating system are shown in Fig. 6 for the temporary capture event with the longest recorded duration. The orbital energy with respect to Mercury is derived from the energy equation of an elliptic orbit (e.g., Danby 1992),

$E_{\text {orb }} \propto E=-\frac{1}{a}=\frac{v^{2}}{k^{2} \mu_{\mathrm{Merc}}}-\frac{2}{\Delta}$,

where $a$ is the hermeocentric distance in AU, $v$ the orbital velocity, $k$ the constant of gravitation, $\mu_{\text {Merc }}$ the mass of Mercury in solar units, and $\Delta$ the distance between Mercury and the object in AU.

The trajectory of a typical group I member is illustrated in Fig. 5. A number of observations can be made for this type of objects. First, the closest Mercury approach generally does not coincide with the lowest hermeocentric orbital energy. Second, $E<0$ for all approaches; thus the resultant gravitational attraction of Mercury on the object is temporarily greater than that of the Sun. Third, one subgroup of objects perform a loop around Mercury in the corotating frame, while others (as in Fig. 5) do not. Fourth, objects may reverse sense of rotation relative to Mercury during approach and temporary capture. For all approaches studied, such events occur when Mercury is close to perihelion.

For group II category objects, at least one complete revolution in the same sense around Mercury is performed (Fig. 6). $60 \%$ of the objects perform all revolutions in the same sense, while the others experience a reversal in Mercury's corotating system. Again, this may be due to the change in the direction of the radial velocity vector of Mercury at aphelion or perihelion passage.

\subsection{Hermeocentric orbits}

The stability region for prograde and retrograde Mercury orbiters was studied by integrating 16 pairs of objects with varying initial pericenter distances and eccentricities. 

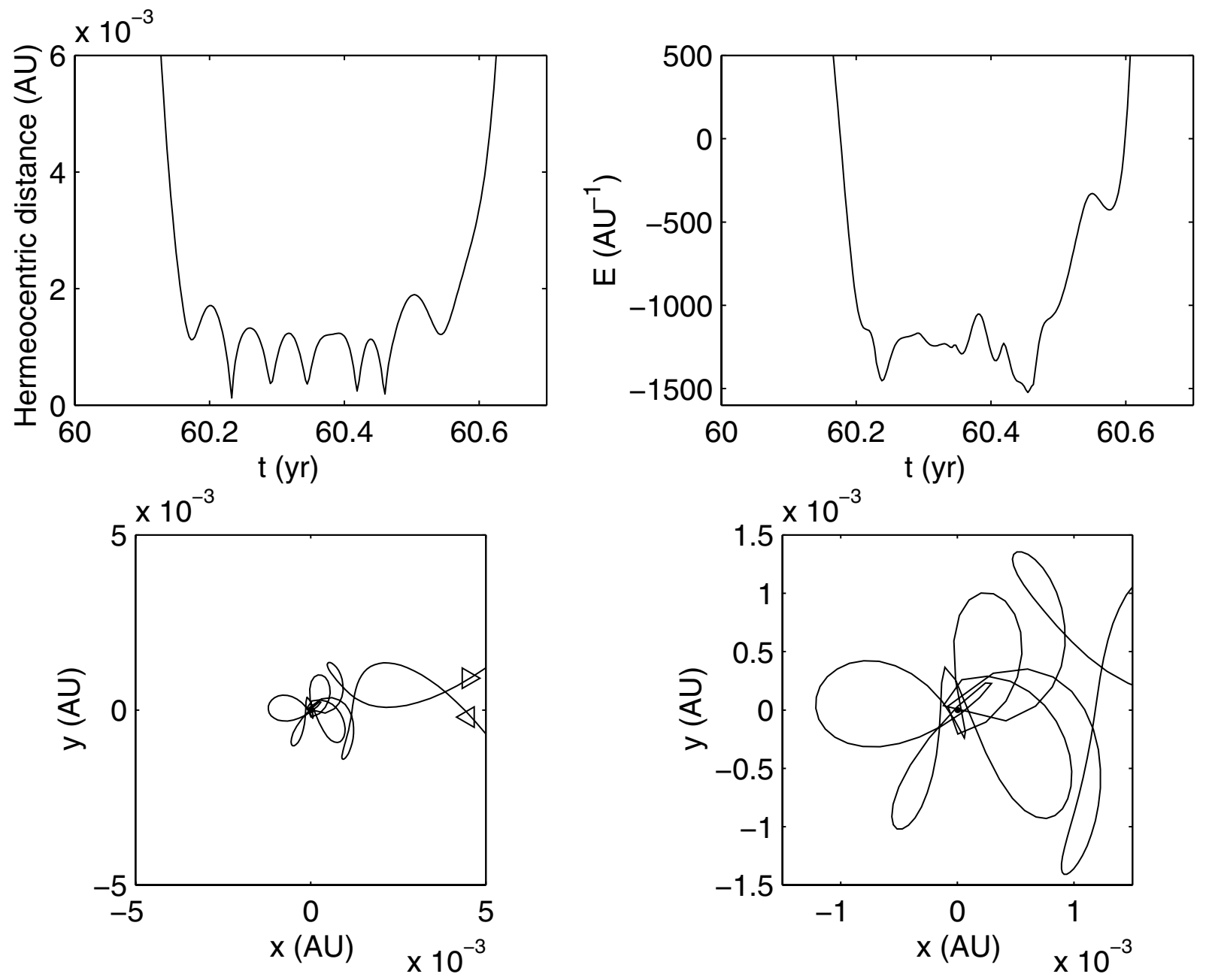

Fig. 6. As in Fig. 5, but for object 853.3. Entry and exit occur through L2 and four complete orbits are performed with three in prograde sense and an intermediate one in retrograde sense. This event is the longest temporary capture found (from the 1226 year long integration 3 ) with a duration of close to two mercurian years (143.5 days). This object is a group II member (cf. Table 2).

To complement the originally identified orbiter (integration 2) an object with identical initial rectangular elements but with a prograde orbit was integrated, as well as objects with prograde and retrograde orbits around Mercury at initial pericenter distances of $15,30,45,60$ and 75 Mercury radii $\left(R_{\text {Merc }}\right)$. The objects were initiated in the direction of the starting position of the original orbiter (coincidentally in the direction of the radius vector of Mercury and L1) and at pericenter with initial velocities obtained from the energy relation for the elliptic orbit. The initial orbital planes were the same for all objects; thus they inherited the inclination of the orbit of the original object ( $\sim 45^{\circ}$ for the duration of the 100 day short-term integration). The orbital eccentricities were varied so that all possible initial combinations of apohermia and perihermia at these five distances were represented, ranging from circular orbits at the five different radii to a highly eccentric pair of orbits with perihermia at $15 R_{\text {Merc }}$ and apohermia at $75 R_{\text {Merc }}$. The initial orbits and evolution of the objects are summarized in Tables 3 and 4 .

As expected, prograde objects are more likely to escape from the hermeocentric orbits than retrograde objects. After 100 days, nine of the sixteen prograde objects (56\%) had escaped while only one (6\%) retrograde body had done so (cf.
Fig. 7 and Table 3). The escaped objects include all prograde orbiters with initial apohermia at $75 R_{\text {Merc }}$ and the retrograde orbiter with the highest initial orbital eccentricity. Of the ten escaped objects, equal numbers left through L1 and L2. The timing of the ejections do not correlate with the mean anomaly of Mercury. Thus, it appears that the twice as strong gravitational attraction from the Sun at perihelion than at aphelion is unimportant in this respect. We also find that some orbits are stabilized with time in that $a$ and/or $e$ slightly decreases (Table 4).

At a time of $4.5 \mathrm{Myr}$, three prograde and four retrograde objects remained in orbit. These were initially occupying the lowest orbits with pericenter distances of $15-45 R_{\text {Merc }}$ and eccentricities $<0.5$. The other objects tended to escape after a very short time, but one retrograde object escaped after $0.78 \mathrm{Myr}$ and the original moon after 2.46 Myr. The hermeocentric orbital evolution is very fast due to strong solar perturbations. The majority of escaped objects subsequently performed close approaches to Mercury in heliocentric orbits. Of the 24 escaped objects, 18 (75\%, of which 11 prograde, 7 retrograde) collided with Mercury within 1.3 Myr. 
Table 3. Orbital parameters in the short-term hermeocentric integration. Columns are, from left: Object designation; initial hermeocentric $a\left(a_{\text {ini }}\right)$ and $e\left(e_{\text {ini }}\right)$; average $a$ and $e$ during hermeocentric orbit, with extreme ranges; average orbital period; minimal and maximal periods (relative to initial $M$ of hermeocentric orbit); duration of hermeocentric orbit ( $P>>$ ); number of revolutions relative to initial $M$ of hermeocentric orbit $\left(N_{\text {orb }}\right)$; rotational sense (prograde/retrograde); mean anomaly of Mercury at time of escape from hermeocentric orbit ( $\left.L_{\text {esc }}\right)$; identity of Lagrangian point traversed at escape from hermeocentric orbit $\left(T_{\mathrm{CO}}\right)$; and time of collision with Mercury. Data from integration 5 (100 day duration). Object $93 \mathrm{r}$ is the originally identified satellite. For object 75-75p, close approaches with Venus are initiated after 1.78 Myr.

\begin{tabular}{|c|c|c|c|c|c|c|c|c|c|c|c|c|c|c|c|}
\hline Object & $\begin{array}{l}a_{\text {ini }} \\
\left(10^{-3} \mathrm{AU}\right)\end{array}$ & $e_{\text {ini }}$ & $\begin{array}{l}\langle a\rangle \\
\left(10^{-3} \mathrm{AU}\right)\end{array}$ & $<e>$ & $\begin{array}{l}<P> \\
\text { (d) }\end{array}$ & $\begin{array}{l}P_{\min } \\
\text { (d) }\end{array}$ & $\begin{array}{l}P_{\max } \\
\text { (d) }\end{array}$ & $\begin{array}{l}t_{\text {orb }} \\
\text { (d) }\end{array}$ & $\begin{array}{l}T_{Q_{\max }} \\
\text { (d) }\end{array}$ & $\begin{array}{l}T_{q_{\min }} \\
\text { (d) }\end{array}$ & $N_{\text {orb }}$ & Sense & $\begin{array}{l}M_{\mathrm{esc}} \\
\left(^{\circ}\right)\end{array}$ & $\bar{L} L_{\mathrm{esc}}$ & $T_{\mathrm{CO}}$ \\
\hline $93 r$ & - & - & $0.704 \pm 0.041$ & $0.412 \pm 0.064$ & 13.86 & 13.0 & 14.8 & $>100$ & - & - & - & $\mathrm{R}$ & - & - & \\
\hline $93 p$ & - & - & $0.673 \pm 0.057$ & $0.488 \pm 0.144$ & 19.95 & 17.80 & 22.6 & 97.6 & 91.8 & 17.6 & 4 & $\mathrm{P}$ & 49.34 & 2 & 17578 \\
\hline $15-15 r$ & 0.245 & 0 & $0.244 \pm 0.000$ & $0.005 \pm 0.001$ & 3.29 & 3.2 & 3.4 & $>100$ & - & - & - & $\mathrm{R}$ & - & - & - \\
\hline $15-15 p$ & 0.245 & 0 & $0.244 \pm 0.001$ & $0.004 \pm 0.002$ & 3.57 & 3.4 & 3.8 & $>100$ & - & - & - & $\mathrm{P}$ & - & - & - \\
\hline $30-15 r$ & 0.367 & 0.333 & $0.369 \pm 0.007$ & $0.385 \pm 0.038$ & 5.89 & 5.6 & 6.2 & $>100$ & - & - & - & $\mathrm{R}$ & - & - & - \\
\hline $30-15 p$ & 0.367 & 0.333 & $0.368 \pm 0.005$ & $0.226 \pm 0.050$ & 6.84 & 6.6 & 7.2 & $>100$ & - & - & - & $\mathrm{P}$ & - & - & - \\
\hline $45-15 r$ & 0.735 & 0.500 & $0.501 \pm 0.018$ & $0.573 \pm 0.056$ & 8.85 & 8.2 & 9.8 & $>100$ & - & - & - & $\mathrm{R}$ & - & - & - \\
\hline $45-15 p$ & 0.735 & 0.500 & $0.505 \pm 0.026$ & $0.250 \pm 0.085$ & 11.63 & 11.0 & 12.4 & $>100$ & - & - & - & $\mathrm{P}$ & - & - & - \\
\hline $60-15 r$ & 0.612 & 0.600 & $0.656 \pm 0.034$ & $0.656 \pm 0.095$ & 12.4 & 10.2 & 14.8 & $>100$ & - & - & - & $\mathrm{R}$ & - & - & - \\
\hline $60-15 p$ & 0.612 & 0.600 & $0.738 \pm 0.193$ & $0.273 \pm 0.141$ & 20.47 & 18.4 & 23.4 & 90.2 & 24.2 & 66.8 & 3 & $\mathrm{P}$ & 19.06 & 1 & 964 \\
\hline $75-15 r$ & 0.735 & 0.667 & $0.872 \pm 0.000$ & $0.874 \pm 0.000$ & 19.50 & 17.4 & 21.6 & 34.8 & 22.0 & 12.4 & 2 & $\mathrm{R}$ & 152.34 & 1 & 1007 \\
\hline $75-15 p$ & 0.735 & 0.667 & - & - & - & - & - & - & - & 20.2 & 0 & $\mathrm{P}$ & 93.41 & 2 & 406817 \\
\hline $30-30 r$ & 0.490 & 0 & $0.482 \pm 0.007$ & $0.043 \pm 0.012$ & 8.56 & 8.2 & 9.0 & $>100$ & - & - & - & $\mathrm{R}$ & - & - & - \\
\hline 30-30p & 0.490 & 0 & $0.476 \pm 0.014$ & $0.034 \pm 0.025$ & 10.51 & 9.8 & 11.4 & $>100$ & - & - & - & $\mathrm{P}$ & - & - & - \\
\hline $45-30 r$ & 0.612 & 0.200 & $0.602 \pm 0.013$ & $0.260 \pm 0.053$ & 11.43 & 10.8 & 11.8 & $>100$ & - & - & - & $\mathrm{R}$ & - & - & - \\
\hline $45-30 p$ & 0.612 & 0.200 & $0.591 \pm 0.022$ & $0.054 \pm 0.079$ & 15.30 & 14.0 & 16.4 & $>100$ & - & - & - & $\mathrm{P}$ & - & - & - \\
\hline $60-30 r$ & 0.735 & 0.333 & $0.725 \pm 0.028$ & $0.486 \pm 0.029$ & 14.33 & 13.2 & 16.2 & $>100$ & - & - & - & $\mathrm{R}$ & - & - & - \\
\hline 60-30p & 0.735 & 0.333 & $0.703 \pm 0.030$ & $0.089 \pm 0.200$ & 20.80 & 19.4 & 22.2 & $>100$ & - & - & - & $\mathrm{P}$ & - & - & - \\
\hline $75-30 \mathrm{r}$ & 0.857 & 0.429 & $0.924 \pm 0.272$ & $0.503 \pm 0.289$ & 19.08 & 15.4 & 23.0 & $>100$ & - & - & - & $\mathrm{R}$ & - & - & - \\
\hline $75-30 p$ & 0.857 & 0.429 & $0.842 \pm 0.065$ & $0.091 \pm 0.130$ & 27.33 & 25.4 & 29.4 & 88.6 & 67.6 & 22.0 & 3 & $\mathrm{P}$ & 12.51 & 1 & - \\
\hline $45-45 r$ & 0.735 & 0 & $0.702 \pm 0.016$ & $0.155 \pm 0.096$ & 14.03 & 12.6 & 15.2 & $>100$ & - & - & - & $\mathrm{R}$ & - & - & - \\
\hline $45-45 p$ & 0.735 & 0 & $0.631 \pm 0.031$ & $0.347 \pm 0.116$ & 17.95 & 16.8 & 20.8 & 91.0 & 86.4 & 19.0 & 4 & $\mathrm{P}$ & 22.33 & 2 & 7155 \\
\hline $60-45 r$ & 0.857 & 0.143 & $0.804 \pm 0.038$ & $0.083 \pm 0.057$ & 16.80 & 15.8 & 17.6 & $>100$ & - & - & - & $\mathrm{R}$ & - & - & - \\
\hline $60-45 p$ & 0.857 & 0.143 & $0.714 \pm 0.074$ & $0.487 \pm 0.153$ & 22.87 & 19.0 & 26.0 & 85.4 & 78.8 & 49.8 & 3 & $\mathrm{P}$ & 359.42 & 2 & 86781 \\
\hline $75-45 r$ & 0.979 & 0.250 & $0.934 \pm 0.053$ & $0.360 \pm 0.074$ & 19.20 & 17.4 & 21.0 & $>100$ & - & - & - & $\mathrm{R}$ & - & - & - \\
\hline $75-45 p$ & 0.979 & 0.250 & $0.833 \pm 0.132$ & $0.492 \pm 0.124$ & 31.20 & 24.4 & 38.0 & 84.6 & 76.8 & 69.6 & 2 & $\mathrm{P}$ & 356.14 & 2 & 436494 \\
\hline $60-60 \mathrm{r}$ & 0.979 & 0 & $0.878 \pm 0.027$ & $0.323 \pm 0.138$ & 19.16 & 16.4 & 21.8 & $>100$ & - & - & - & $\mathrm{R}$ & - & - & - \\
\hline 60-60p & 0.979 & 0 & $0.739 \pm 0.113$ & $0.677 \pm 0.157$ & 18.68 & 14.6 & 24.8 & $>100$ & - & - & - & $\mathrm{P}$ & - & - & - \\
\hline $75-60 r$ & 1.102 & 0.111 & $0.982 \pm 0.017$ & $0.174 \pm 0.271$ & 22.05 & 19.4 & 23.6 & $>100$ & - & - & - & $\mathrm{R}$ & - & - & - \\
\hline $75-60 p$ & 1.102 & 0.111 & - & - & - & - & - & 17.2 & 0 & $\mathrm{P}$ & 81.14 & 1 & 23902 & & \\
\hline $75-75 r$ & 1.224 & 0 & $1.037 \pm 0.033$ & $0.521 \pm 0.144$ & 23.65 & 18.4 & 29.2 & $>100$ & - & - & - & $\mathrm{R}$ & - & - & - \\
\hline $75-75 p$ & 1.224 & 0 & - & - & - & 5.5 & - & 9.8 & 0 & $\mathrm{P}$ & 50.85 & 1 & & & \\
\hline
\end{tabular}


Table 4. Orbital parameters in the long-term hermeocentric integration. Notes: Columns are, from left: Object designation; initial hermeocentric $a$ and $e$; average $a$ with extreme range; duration of hermeocentric orbit; rotational sense (prograde/retrograde); notes or end fate ("CO" indicates collision with Mercury). Data from integration 5 for situation at $t=4.54$ Myr. Values of $<a>$ are for the initial $75 \mathrm{kyr}$ of the integration, unless otherwise indicated in the "Note" column. Object $93 \mathrm{r}$ is the originally identified satellite.

\begin{tabular}{|c|c|c|c|c|c|c|}
\hline Object & $\begin{array}{r}a_{\text {ini }} \\
(\mathrm{AU}) \\
\end{array}$ & $e_{\text {ini }}$ & $\begin{array}{r}<a> \\
(\mathrm{AU}) \\
\end{array}$ & $\begin{array}{r}t_{\text {orb }} \\
(\mathrm{yr}) \\
\end{array}$ & Sense & Note \\
\hline $93 r$ & - & - & $0.000742 \pm 0.000207$ & $2.46 \times 10^{6}$ & $\mathrm{R}$ & \\
\hline $15-15 r$ & 0.000245 & 0 & $0.000245 \pm 0.000034$ & $>4.54 \times 10^{6}$ & $\mathrm{R}$ & \\
\hline $15-15 p$ & 0.000245 & 0 & $0.000247 \pm 0.000030$ & $>4.54 \times 10^{6}$ & $\mathrm{P}$ & \\
\hline ibid & & & $0.000244 \pm 0.000002$ & & & $1.81<t(\mathrm{Myr})<4.54$ \\
\hline $30-15 r$ & 0.000367 & 0.333 & $0.000371 \pm 0.000079$ & $>4.54 \times 10^{6}$ & $\mathrm{R}$ & \\
\hline $30-15 p$ & 0.000367 & 0.333 & $0.000374 \pm 0.000088$ & $>4.54 \times 10^{6}$ & $\mathrm{P}$ & \\
\hline $45-15 r$ & 0.000490 & 0.500 & $0.000515 \pm 0.000230$ & $>4.54 \times 10^{6}$ & $\mathrm{R}$ & \\
\hline $45-15 p$ & 0.000490 & 0.500 & $0.000516 \pm 0.000131$ & 2420 & $\mathrm{P}$ & $\mathrm{CO}$ at $141 \mathrm{kyr}$ \\
\hline $60-15 r$ & 0.000612 & 0.667 & - & 4.6 & $\mathrm{R}$ & $\mathrm{CO}$ at $1.29 \mathrm{Myr}$ \\
\hline $30-30 \mathrm{r}$ & 0.000490 & 0 & $0.000490 \pm 0.000076$ & $>4.54 \times 10^{6}$ & $\mathrm{R}$ & \\
\hline ibid & & & $0.000491 \pm 0.000126$ & & & $1.81<t(\mathrm{Myr})<2.21$ \\
\hline $30-30 p$ & 0.000490 & 0 & $0.000478 \pm 0.000018$ & $>4.54 \times 10^{6}$ & $\mathrm{P}$ & \\
\hline $45-30 \mathrm{r}$ & 0.000612 & 0.200 & $0.000634 \pm 0.000188$ & $7.83 \times 10^{5}$ & $\mathrm{R}$ & $\mathrm{CO}$ at $3.04 \mathrm{Myr}$ \\
\hline ibid & & & $0.000598 \pm 0.000181$ & & & $51.6<t(\mathrm{kyr})<78.2$ \\
\hline $45-30 p$ & 0.000612 & 0.200 & - & 4.8 & $\mathrm{P}$ & $\mathrm{CO}$ at 92 years \\
\hline $60-30 \mathrm{r}$ & 0.000736 & 0.333 & - & 52 & $\mathrm{R}$ & $\mathrm{CO}$ at $214 \mathrm{kyr}$ \\
\hline $60-30 p$ & 0.000736 & 0.333 & - & 0.50 & $\mathrm{P}$ & $\mathrm{CO}$ at 2358 years \\
\hline $75-30 \mathrm{r}$ & 0.000857 & 0.429 & - & 0.54 & $\mathrm{R}$ & \\
\hline $45-45 r$ & 0.000736 & 0 & - & 56 & $\mathrm{R}$ & \\
\hline $60-45 r$ & 0.000857 & 0.143 & - & 21 & $\mathrm{R}$ & $\mathrm{CO}$ at 3490 years \\
\hline $75-45 r$ & 0.000979 & 0.250 & - & 15 & $\mathrm{R}$ & $\mathrm{CO}$ at $16.6 \mathrm{kyr}$ \\
\hline $60-60 \mathrm{r}$ & 0.000979 & 0 & - & 3.4 & $\mathrm{R}$ & $\mathrm{CO}$ at 478 years \\
\hline $60-60 p$ & 0.000979 & 0 & - & 0.46 & $\mathrm{P}$ & $\mathrm{CO}$ at $200 \mathrm{kyr}$ \\
\hline $75-60 \mathrm{r}$ & 0.001102 & 0.111 & - & 1.7 & $\mathrm{R}$ & \\
\hline $75-75 r$ & 0.001224 & 0 & - & $>0.27,<1.29$ & $\mathrm{R}$ & $\mathrm{CO}$ at 472 days \\
\hline
\end{tabular}

One object (representing $4 \%$ of the escaped number), 75-75p, acquired a Venus crossing orbit and has been integrated for 4.5 Myr. It was initiated within the Hill sphere of Mercury but was not bound (Table 3). At $1.78 \mathrm{Myr}$ the object attained a Venus crossing orbit due to a capture in $v_{1}$ which raised $e$ from 0.25 to 0.6 . Despite a total of 10351 passages within the Hill sphere of Venus during a timeframe of less than $2 \mathrm{Myr}$ for this object, no collision with Venus occurs, as well as no consistent pumping of $a$ to allow a circularization of the orbit. The eccentricity is high at $0.5-0.7$ during most of the integrated period, which causes maximal aphelion distances of $0.82 \mathrm{AU}$, but the object is still far short of becoming Earth crossing.

\section{Number of objects $\mathcal{N}$ with Mercury-like orbits}

In this section we attempt to estimate the number of current objects residing in Mercury-like orbits, except for the planet itself. The three different methods used provide only order of magnitude estimates, but all indicate an upper limit to the num- ber of $<1$; thus, no object is likely to be found at present. This estimate may or may not be relevant to the number of hermeocentric objects. If the time scale against perturbations of close near-circular orbits to Mercury is similar to the age of the solar system, there is a high probability that hermeocentric objects, albeit small, exist today. From the present work, we can only conclude that the time scale for hermeocentric objects with semimajor axes $<30$ Mercury radii is at least as large as the time scale of gravitational scattering for Mercury-like heliocentric orbits (a few million years), and most probably significantly larger. However, the discussion below on the probable present number of objects is restricted to heliocentric orbits.

Objects with semimajor axes close to that of Mercury ( $a=$ $0.3871 \mathrm{AU}$ ) can be removed comparatively fast from this region, primarily due to collisions. The expected dynamical lifetime of such an object is of the order of 5 Myr or less (e.g., Evans \& Tabachnik 1999; Gladman et al. 1997). Unless the original number of objects was considerably larger than current estimates allow, no asteroid originally present is likely to have survived over the age of the solar system. Any objects 

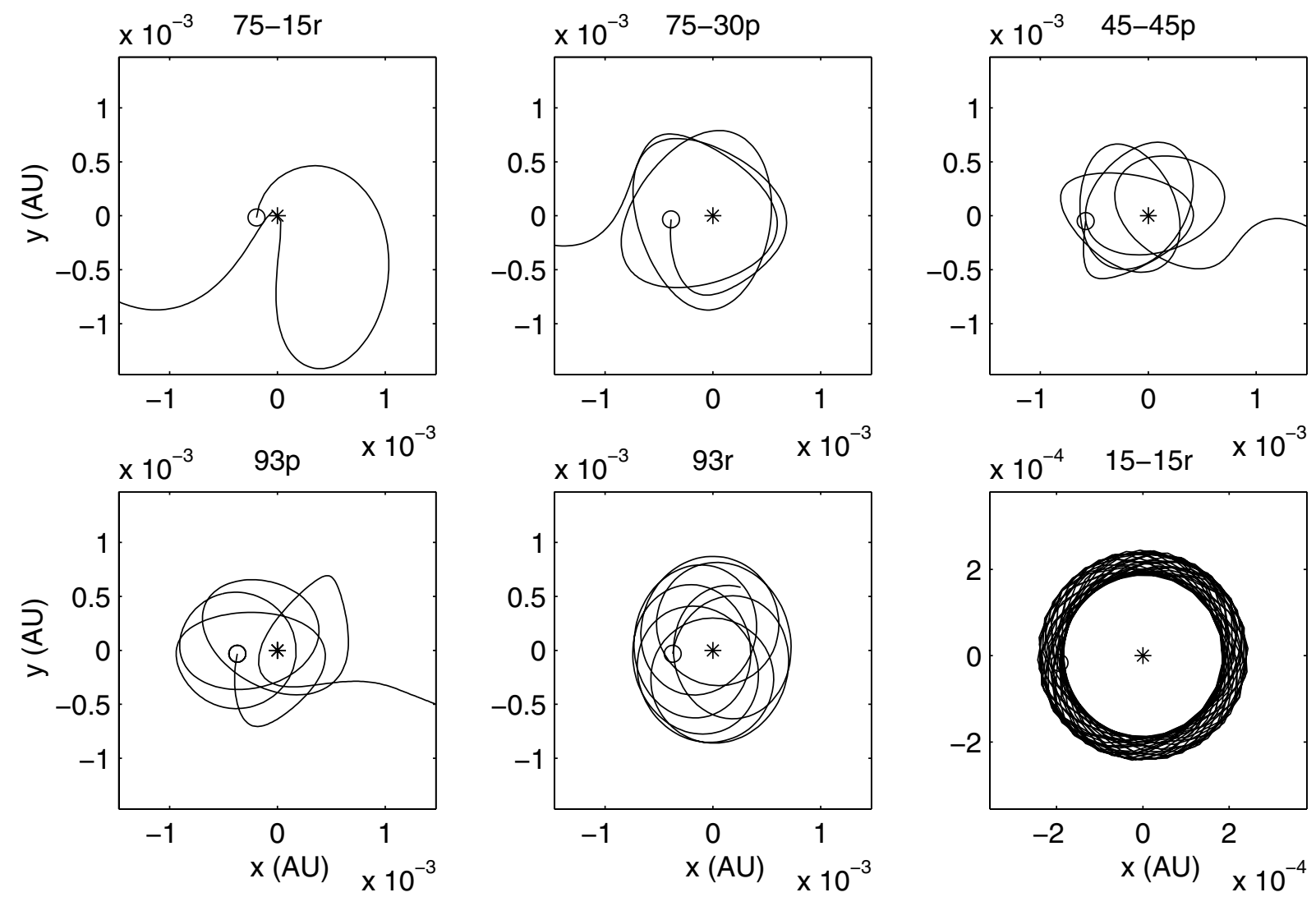

Fig. 7. 100 day trajectories of selected objects from the short-term hermeocentric integration. Orbits are shown in the mercurian corotating reference system with Mercury at the center (asterisk) and the sun on the negative $x$ axis, projected on the osculating $X-Y$ plane of Mercury. The initial location of the object is marked with a circle. Orbits are increasingly stable in row-wise order from upper left, with durations until escape of 34.8 days, 88.6 days, 91.0 days, 97.6 days, 2.46 Myr and >4.54 Myr (note scale change in last panel). The pericenter longitude is rapidly rotating for all objects. Escape trajectories pass through either L1 (sunward) or L2, which are located on the $y$ axis at $x \sim \pm 1.5 \times 10^{-3}$ AU.

which are present in the region today must therefore have originated somewhere else and been perturbed into their present orbits due to one of several possible mechanisms, e.g. thermal forces (Yarkovsky or Poynting-Robertson effects), orbital resonances, collisional events or close encounters with the planets. Several sources of origin for objects with Mercury-like orbits are possible, including the asteroid main belt, ejecta from collisional events in the inner solar system, near-Earth asteroids, extinct comets, the proposed Vulcanoid belt population, or even objects originally in the 1:1 Mercury resonance or hermeocentric orbit.

No natural asteroids are known with orbits totally inside that of the Earth. However, about 200 Earth crossing objects with orbital semimajor axis less than that of the Earth (Aten asteroids) are known. About $40 \%$ of these, as well as $11 \%$ of the presently known population of about 1000 Apollo asteroids, cross the orbit of Mercury. Other members of these groups will eventually develop Mercury crossing orbits, as is the case also for many comets.

Inside the orbit of Mercury a proposed class of asteroids known as the Vulcanoids may constitute a dynamically stable population (e.g., Vokrouhlický et al. 2000 and references therein). According to the models of iron-silicate fractionation in the solar nebula (Weidenshilling 1978) and a late giant impact (Cameron et al. 1988; Benz et al. 1988; Wetherill 1988) for the geological and geophysical evolution of Mercury, the Vulcanoids may have extended the period of late heavy bombardment of the planet by up to 1 Gyr (Leake et al. 1987). In this process such objects may have also been dissipatively captured by Mercury. Dynamical studies have shown that objects on near-circular orbits with semimajor axes between approximately $0.09 \mathrm{AU}$ and $0.21 \mathrm{AU}$ may be stable over the age of the solar system (Evans \& Tabachnik 1999). However, due to collisional evolution (Stern \& Durda 2000) and the thermal Poynting-Robertson and Yarkovsky forces (Vokrouhlický et al. 2000), this region may have been depleted long ago and thus presently contain very few kilometer-sized objects.

\subsection{Estimate of $\mathcal{N}$ from the number of near-Earth asteroids}

Many estimates of the number of objects whose orbits pass in the vicinity of the Earth have been made (e.g., Shoemaker et al. 1979; Rabinowitz 1994; Rabinowitz et al. 1994, 2000; Bottke et al. 2000; Harris 2000; Werner et al. 2002). Their total number is still very uncertain and disputed, but a reasonable population size is 500-2000 objects larger than $d=1 \mathrm{~km}$ (roughly corresponding to absolute magnitude $H<18$ ). In the method used here to obtain a rough estimate of the number of near-Earth Asteroids (NEA's) with Mercury-like orbits and sizes larger 
than one kilometer, we compare the total volume encompassed by the perihelion distances of the NEA's to that of objects with Mercury-like orbits, and derive an estimate of the latter from a statistical argument based on the range of orbital elements of the known NEA population. We assume that the total number is $\sim 2000$ and that their volume number density is constant in the perihelion range $0.25<q<1.3 \mathrm{AU}$ and ignore several complicating factors, e.g. resonances related to the major planets, thermal forces and the possibility that the number density decreases away from the main belt. The volume of the spherical shell enclosing the perihelia of the NEA's may now be compared to other spherical shells closer to the Sun.

Taking into account the fact that the large free eccentricity of Mercury attains extreme values within a time period of 1 Myr (Leake et al. 1987) and effectively increases the volume of the Mercury-enclosing shell by a few times, the most realistic estimate of the present number of objects that are larger than $1 \mathrm{~km}$ and have Mercury-like orbits is found to be $\mathcal{N} \sim 10^{-3}$.

\subsection{Estimate of $\mathcal{N}$ from objects with Earth- and Mars-like orbits}

An estimate of the number of objects in Mercury-like orbits may be obtained from the known number of objects in orbits which are very similar to those of Mars and the Earth. By excluding consideration of dynamical differences an independent, albeit very approximate, number is obtained from a perihelion distance argument. Six Mars Trojans (cf. http://cfa-www.harvard.edu/iau/lists/MarsTrojans.html) and one object in the 1:1 mean motion resonance with the Earth (2002 $\mathrm{AA}_{29}$; Connors et al. 2002) are presently known, constituting $\sim 0.3 \%$ of the NEA population. Defining a current Mercury-like orbit with the criterion that the semimajor axis is in the range $0.365-0.415 \mathrm{AU}$, this shell-like volume corresponds to $0.05 \%$ of the spherical volume encompassed by the perihelion distances of NEA's. Thus, $\sim 1$ objects should have perihelion distances in the shell, of which $0.3 \%$ or $\mathcal{N} \sim 10^{-4}$ objects statistically have Mercury-like orbits.

\subsection{Estimate of $\mathcal{N}$ from solar nebula constraints}

Weidenschilling (1978) showed in his model for iron/silicate fractionation during the accretion of Mercury that objects remaining within the orbit of the planet after the dissipation of the solar nebula were probably larger than $10 \mathrm{~km}$. The total initial mass of material remaining within Mercury's perihelion after the dissipation of the nebula was estimated to $\sim 10 \%$ of Mercury's mass, or $3 \times 10^{22} \mathrm{~kg}$. Assuming that these objects had similar bulk densities as Mercury and diameters of $10 \mathrm{~km}$, a total of $1.5 \times 10^{7}$ objects would have been initially located within the orbit of Mercury. Orbits with $a$ larger than $0.25 \mathrm{AU}$ would cause objects to become Mercury crossing and be depleted over a period of approximately $10^{9}$ years (Leake et al. 1987). Applying this exponential half-life time scale predicts that $10^{-9}$ of the initial population, $\sim 10^{-2}$ objects, should remain in the region $a=0.25-0.35 \mathrm{AU}$ at the age of the so- lar system. For an equivalent mass of $1 \mathrm{~km}$ size objects, collisionally derived from the larger planetesimal bodies following the dissipation era, this number increases to $\sim 10$. However, the collisional destruction process is not fully efficient in creating kilometer-sized objects, and will additionally produce smaller objects which will be removed by further collisions with each other and with Mercury, as well as by thermal forces. Thus, the best estimate of $\mathcal{N}$ remains $<1$.

\section{Possibility of detecting a hermeocentric object}

The possibility of detecting an object which is in orbit around Mercury or passing through its Hill sphere depends to first order on its size, to second order on its albedo. The most reasonable assumption is that potential objects are strongly matured by the space weathering process and have the same reflectivity and integral phase function as Mercury.

The most favorable observational geometry for a search of mercurian satellites is at maximal solar elongation points of Mercury's orbit, particularly when near its aphelion, and at true astronomical night for best signal-to-noise ratio. A spherical, half-illuminated, object $1 \mathrm{~km}$ in size would have a $V$-band magnitude of $\sim 20$ at this geometry. This is within reach of a 2.5-m class telescope using an unfiltered 5 second CCD integration at seeing conditions of $1.5^{\prime \prime}$ at an air mass of six.

Considering the practical detectability of a typical object with a single suitable telescope, the volume within $150000 \mathrm{~km}$ from Mercury, equivalent to a radius of $\sim 4^{\prime}$, should preferably be searched three times per darktime window $\left(<1 \mathrm{~h} \mathrm{day}^{-1}\right)$ for a period of at least a few days. This ensures observational coverage of a significant part $(\gtrsim 1 / 4)$ of the trajectory around Mercury to obtain approximate orbital elements. Assuming the worst possible observational geometry, the orbit is seen nearly edge-on, and the object spends $\gtrsim 1$ day twice per revolution at an angular distance $<1^{\prime}$ from Mercury during which detection is difficult. Assuming an average angular orbital velocity of $21^{\circ}$ day $^{-1}$, the apparent sky motion of the object relative to Mercury is $3^{\prime \prime} \mathrm{h}^{-1}$ at the most probable orbital position, a motion that should be detectable within a one hour timeframe. For the part of the orbit that the object spends at the apparent turning points, there is a two day period when the hourly motion falls below the detectability limit, and daily astrometry will have to be used for the orbital solution.

\section{Discussion and conclusions}

Gladman (1997) performed numerical simulations of asteroid main belt particles near and within orbital resonances to investigate their orbital evolution and dynamical lifetimes. Of relevance to this work is the fact that particles injected into $v_{6}$ (for Vesta group asteroids) and the 3:1 mean motion resonance with Jupiter (for the Maria group) also have non-zero probabilities of colliding with Venus; a small fraction ( $\sim 2 \%$ and $\sim 0.5 \%$ respectively) are shown to end this way. For these types of objects, the characteristic time scale for development of Venus crossing orbits is less than $2 \mathrm{Myr}$.

The integrations of Gladman et al. (1997) indicate that orbital types similar to those of objects presented in this study 
(i.e., Venus crossing orbits from originally Mercury-like orbits) may be attained by evolved main belt asteroids. Thus, there may be ways to transfer particles to the inner solar system via secular resonances, particularly $v_{6}$ and similar pericenter resonances of the inner planets, and thus, through the reversibility of motion, transport mercurian meteorites to the Earth. While we do not find evidence in the present work for the action of $v_{6}$ on any integrated particle due to their small semimajor axes (consistent with the results of Michel \& Froeschle 1997), secular resonances (probably non-linear and possibly higher-order combinations of $v_{12}, v_{2}$ and $v_{5}$ ) are shown to be able to force $e$ to high values to produce Venus crossing orbits. Further gravitational scattering by Venus would then increase $a$ to make such objects available for capture by $v_{6}$.

The orbital encounter scenario for two light particles in orbits of different radii around a massive primary was studied in detail by Petit \& Henon (1986). They classified the possible encounter orbits of the satellites into different families and showed that these were determined by the value of the difference in radius of their orbits. These families include temporary capture and horseshoe orbits.

Namouni (1999) studied the dynamics of objects in 1:1 mean motion commensurabilities and the stability to external secular perturbations, and found that for the case of Mercury, such orbits with high inclinations and eccentricities may be stable with respect to secular perturbations due to Mercury's large distance from Venus.

Dvorak (1986) reviews the studies made on purely gravitational capture of comets and asteroids by the planets and examines the process theoretically. The conclusion is that in the circular and elliptical restricted cases and the $n$-body problem, permanent capture can only occur when non-gravitational forces are involved. If not, only a temporary capture is possible, and only for close approaches.

Horedt (1976) studied the orbital evolution of objects with significant non-zero velocities at L1 in a corotating system centered on a planet with an eccentric heliocentric orbit. He concluded that satellites captured with small relative velocities in L1 can only gain direct orbits with small inclinations and semimajor axes in the range $0.33 v<a<0.46 v$, where $v \sim$ $(\mu / 3)^{1 / 3}$ and $\mu \ll 1$ and that, through the reversibility of motion, a satellite fulfilling these criteria must also escape with low velocity through $\mathrm{L} 1$.

Our finding that retrograde satellite orbits are more stable than prograde is in accordance with other dynamical studies (e.g., Henon 1969). Horedt (1976) investigated the problem of satellite capture from a heliocentric to a planetocentric orbit in the coplanar circular restricted three-body problem with constant masses and no energy dissipation. He found that direct satellites are stable for semimajor axes in the osculating planetocentric orbit of about $v / 2$ in units of the Sun-planet distance. A retrograde satellite is stable for twice as large a range of planetocentric distances, and in general the stability zone extends out to semimajor axes equal to the separation of the planet and its L1 point. For Mercury with $\mu=1 / 6023600$, direct stable satellites would then be possible within $90000 \mathrm{~km}$ of the planet, while for retrograde orbits the figure is $180000 \mathrm{~km}$ but may be larger for certain initial conditions.
Rawal (1986) performed an analytical study of the stability of mercurian satellites based on the coplanar restricted three-body problem and the location of stationary direct and retrograde orbits. For a retrograde orbit, he found that the stability zone extends from the geostationary orbit (inferior of which the satellite will eventually spiral inside the Roche radius) at $225000 \mathrm{~km}$ to the limiting retrograde orbit (outside of which the gravitational perturbations of the Sun will cause it to escape) at $252700 \mathrm{~km}$ from Mercury's center. For a prograde orbit, the limiting radius falls inside the stationary orbit, and thus no stable prograde objects should exist.

In the present numerical study we find hermeocentric retrograde orbits that are stable for $>4.5 \mathrm{Myr}$ for initial semimajor axes $<30$ Mercury radii $(73000 \mathrm{~km})$ and eccentricities $<0.5$, while for prograde objects only circular orbits are comparatively stable. If such objects are stable for time scales comparable to the age of the solar system, they may still exist. Optical searches within a radius of at least $150000 \mathrm{~km}$ from Mercury (corresponding to $4^{\prime}$ at maximal elongation from the Sun) are thus motivated and should be able to detect objects of $1 \mathrm{~km}$ size or smaller. A search for mercurian satellites on Mariner $10 \mathrm{im}-$ ages has shown that the existence of objects larger than $5 \mathrm{~km}$ within 30 Hermeocentric radii is improbable (Murray et al. 1974).

Surveys at solar elongations of less than $15^{\circ}$ should also be attempted to set more usable constraints on the number of objects in the putative Vulcanoid population. However, in addition to being observationally more difficult than satellite searches, they are considerably more time-consuming as a large sky area must be covered. Previous searches for Vulcanoid members have yielded only poor constraints to the number of such objects. Leake et al. (1987) determined that no appreciable population of large $(>50 \mathrm{~km})$ bodies interior to Mercury could exist, while Durda et al. (2000) estimated that at most 180042000 objects larger than $1 \mathrm{~km}$ are currently present in the dynamically stable region $0.07-0.21$ AU from the Sun. Recent theoretical studies have estimated the present number to no more than a few hundred larger than $1 \mathrm{~km}$ (Stern \& Durda 2000; Vokrouhlický et al. 2000).

As for objects existing in Mercury-like orbits (as defined in Sect. 2), we obtain estimates based on the population of near-Earth asteroids, objects in the 1:1 mean motion resonances with the Earth and Mars, and Weidenschilling's (1978) model of the solar nebula, that constrain the present number of such $1 \mathrm{~km}$ size objects to $<1$. As we do find that hermeocentric and 1:1 Mercury mean motion resonance orbits which are dynamically stable for long time periods do exist, we can however not rule out the possibility that this number is unity or larger.

Of specific interest in the present study is the conclusion that very close passages to Mercury are needed for significant gravitational scatter of the approaching particle. We find that a typical encounter with Mercury results in a change of the semimajor axis of the particle orbit of $\Delta a \sim 0.01 \mathrm{AU}$ (a similar result was obtained by Leake et al. 1987). Thus, collisional ejecta resulting from an impact on Mercury which receive velocities slightly in excess of the escape velocity and are sent into heliocentric Mercury-like orbits will very likely be sub- 
jected to successive close approaches with a high probability of reaccretion.

To estimate the importance of this effect on the evolution of the early mercurian surface we follow the approach of Lewis (1995) on the topic of regolith formation on the Martian moons. In this model, collisionally ejected material preferentially fills a toroidal-shaped volume centered on the planet's orbit. An object ejected with low velocity into an orbit similar to Mercury's will likely be trapped in the 1:1 resonance which would result in a time scale to homogeneously fill the torus of about $\sim 80$ years. Comparing with the scenario of similar masses ejected at an impact on Mercury and on Phobos, whose regolith has been completely ejected and reassembled a large number of times over the age of the solar system, the toroidal density in Phobos' orbit is only about 100 times greater than for Mercury.

Thus, the effect of secondary impacts on Mercury through sweep-up of heliocentric particles may have been important for regolith formation during the first $1 \mathrm{Gyr}$ when Vulcanoid population objects possibly impacted its surface (Leake et al. 1987) (and which due to their low, $\sim 20 \mathrm{~km} \mathrm{~s}^{-1}$, impact velocities would not have contributed significantly to the heliocentric debris material). Initially the process may have resulted in a crater size-frequency distribution that was unique in the solar system and skewed towards an over abundance of small craters, due to both the low relative velocities and the small size of escaped secondary debris particles. No such tendency in the crater size-frequency distribution is apparent however for craters in the size range $10-40 \mathrm{~km}$ (Strom \& Neukum 1988) when comparing mercurian intercrater plains and lunar highlands. This may be due to intercrater plains emplacement which preferentially obliterated small craters. The impact record for such small crater sizes from the first Gyr of crustal evolution on Mercury is thus not likely to be presently available for study in the paleogeologic stratigraphic record.

Acknowledgements. The anonymous referee is thanked for valuable comments which improved the paper.

\section{References}

Benz, W., Slattery, W. L., \& Cameron, A. G. W. 1988, Icarus, 74, 516 Bottke, W. F., Jedicke, R., Morbidelli, A., et al. 2000, Science, 288, 2190

Burbine, T. H., McCoy, T. J., Nittler, L. R., et al. 2002, Met. Planet. Sci., 37, 1233

Cameron, A. G. W., Benz, W., Fegley, B., Jr., \& Slattery, W. L. 1988, The strange density of Mercury - Theoretical considerations, in Mercury, ed. F. Vilas, C. R. Chapman, \& M. S. Matthews (Tucson: University of Arizona Press), 692

Campins, H., Davis, D. R., Weidenschilling, S. J., \& Magee, M. 1996, Searching for Vulcanoids, in Completing the Inventory of the Solar System, ed. T. W. Rettig, \& J. M. Hahn (San Fransisco: Astron. Soc. of the Pacific), ASP Conf. Ser., 107, 85

Connors, M., Chodas, P., Mikkola, P., et al. 2002, Met. Planet. Sci., 37,1435

Danby, J. M. A. 1992, Fundamentals of Celestial Mechanics, 2nd ed. (Richmond, Virginia: Willmann-Bell, Inc.)
Durda, D. D., Stern, S. A., Colwell, W. B., et al. 2000, Icarus, 148, 312

Dvorak, R. 1986, On the capture origin of the satellites of the solar system, in Asteroids, Comets, Meteors II, ed. C.-I. Lagerkvist, B. A. Lindblad, H. Lundstedt, \& H. Rickman (Uppsala: Astronomiska observatoriet), 165

Evans, N. W., \& Tabachnik, S. 1999, Nature, 399, 41

Everhart, E. 1985, An efficient integrator that uses Gauss-Radau spacings, in Dynamics of Comets: Their Origin and Evolution, ed. A. Carusi, \& G. B. Valsecchi (Dordrecht: Reidel, Astrophysics and Space Science Library), 115, 185

Gladman, B. J., Burns, J. A., Duncan, M., Lee, P., \& Levison, H. F. 1996, Science, 271, 1387

Gladman, B. J., Migliorini, F., Morbidelli, A., et al. 1997, Science, 277,197

Harris, A. W. 2000, Bull. Amer. Astron. Soc., vol. 32

Henon, M. 1969, A\&A, 1, 223

Horedt, G. P. 1976, AJ, 81, 675

Larsen, D. G., Holdaway, R., et al. 2001, The Astronomical Almanac 2001 (Washington: US Government Printing Office), E3

Leake, M. A., Chapman, C. R., Weidenschilling, S. J., Davis, D. R., \& Greenberg, R. 1987, Icarus, 71, 350

Lewis, J. S. 1995, Physics and Chemistry of the Solar System (Academic Press), 363

Love, S. G., \& Kiel, K. 1995, Meteoritics, 30, 269

Melosh, H. J., \& Tonks, W. B. 1993, Meteoritics, 28, 398

Michel, P. 1997, Icarus, 129, 348

Michel, P., \& Thomas, F. 1996, A\&A, 307, 310

Michel, P., \& Froeschlé, Ch. 1997, Icarus, 128, 230

Murray, B., Belton, M., Danielson, G., et al. 1974, Science, 185, 170

Namouni, F. 1999, Icarus, 137, 293

Petit, J.-M., \& Henon, M. 1986, Icarus, 66, 536

Rabinowitz, D. 1994, Icarus, 111, 364

Rabinowitz, D., Bowell, E., Shoemaker, E., \& Muinonen, K. 1994, The population of Earth-crossing asteroids, in Hazards due to Comets and Asteroids, ed. T. Gehrels (Tucson: University of Arizona Press), 285

Rabinowitz, D. 1997, Icarus, 127, 33

Rabinowitz, D., Helin, E., Lawrence, K., \& Pravdo, S. 2000, Nature, 403, 165

Rawal, J. J. 1986, Earth Moon and Planets, 36, 135

Rickman, H., \& Malmort, A. M. 1981, A\&A, 102, 165

Shoemaker, E. M., Williams, J. G., Helin, E. F., et al. 1979, in Asteroids, ed. T. Gehrels (Tucson: University of Arizona Press), 253

Stern, S. A., \& Durda, D. D. 2000, Icarus, 143, 360

Strom, R. G., \& Neukum, G. 1988, in Mercury, ed. F. Vilas, C. R. Chapman, \& M. S. Matthews (Tucson: University of Arizona Press), 336

Vokrouhlický, D., Farinella, P., \& Bottke, W. F. 2000, Icarus, 148, 147 Werner, S. C., Harris, A. W., Neukum, G., et al. 2002, Icarus, 156, 287

Wetherill, G. W. 1984, Meteoritics, 19, 1

Wetherill, G. W. 1988, Accumulation of Mercury from planetesimals, in Mercury, ed. F. Vilas, C. R. Chapman, \& M. S. Matthews (Tucson: University of Arizona Press), 670

Weidenschilling, S. J. 1978, Icarus, 35, 99

Williams, J. G., \& Faulkner, J. 1981, Icarus, 46, 390

Zhao, Z.-Y., \& Liu, L. 1992, Icarus, 100, 136 